\title{
Effect of ionospheric depth on the ionospheric feedback instability: cutoff and subsequent $E_{\|}$modes
}

\author{
R. Cosgrove and R. Doe \\ Center for Geospace Studies, SRI International, Menlo Park, CA, USA
}

Received: 1 April 2010 - Revised: 21 August 2010 - Accepted: 2 September 2010 - Published: 30 September 2010

\begin{abstract}
The ionospheric feedback instability (IFI), which involves feedback between ionospheric modifications and waves reflected off the magnetosphere, has up to this point been analyzed in terms of field line integrated (FLI) ionospheric quantities, that is, with the assumption that the ionospheric thickness can be ignored. In this work we test this assumption by solving the two-fluid equations for a representative ionospheric slab of finite thickness. We find that the results are for the most part incompatible with a description in terms of FLI quantities, and that their use can easily lead to an order of magnitude overestimation of the growth rate. This occurs because the first eigenmode, which is the one compatible with an FLI description, is cutoff above a certain frequency, leaving only higher order modes with wavelengths along $\boldsymbol{B}$ that are subsumed by the slab. Taking the results at face value, the parallel electric fields associated with the higher order modes are a possible contributor to electron heating and plasma structure in the E-region ionosphere.
\end{abstract}

Keywords. Ionosphere (Auroral ionosphere; Ionospheremagnetosphere interactions; Plasma waves and instabilities)

\section{Introduction}

Charge separation in the ionosphere launches an Alfvén wave into the magnetosphere that transmits energy along the lines of the geomagnetic field, and reflects back to the ionosphere, where if the phase of the reflected wave is correct the initial charge separation will be reinforced, such that there is positive feed back and an instability. This phenomena, which is still for the most part a theoretical construct, is referred to as the ionospheric feedback instability (IFI) Atkinson (1970) is generally credited with introducing this idea; he suggested

Correspondence to: R. Cosgrove (russell.cosgrove@ sri.com) the process as a model for the formation of auroral arcs. Sato (1978) gave perhaps the cleanest development of the basic concept, solving the eigenvalue problem of a driven ionospheric layer satisfying field-line-integrated (FLI) continuity equations in charge and number density, and loaded by a complex admittance representative of a passive magnetospheric load. However, Sato (1978) did not attempt any model of the actual magnetospheric load. Trakhtengertz and Feldstein (984), and Lysak (1986, 1991) added models of such, in varying degrees of complexity. Numerical simulations of the instability development have been performed more recently (e.g., Streltsov and Lotko, 2003, 2004; Chaston et al., 2002; Pokhotelov et al., 2004). In the present work we examine the assumption, made in all previous works, that the ionosphere can be reasonably approximated by a thin layer - we find that it cannot. We do this by performing a realistic computation of the complex magnetospheric admittance (using transmission line theory), and applying it to give a boundary condition at the top of a finite thickness ionosphere (described by 2-D two-fluid equations perpendicular and parallel to the magnetic field), and then solving the eigenvalue problem for the growth rates of the eigenmodes.

The theory and modeling of the ionospheric feedback instability (IFI) has, up to this point, assumed that the ionospheric thickness can be ignored; the ionosphere is represented solely by field line integrated quantities. Trakhtengertz and Feldstein (1984) have given a partial discussion of the limitations of this assumption. The assumption is based on the idea that the conductivity in the direction parallel to the magnetic field $\boldsymbol{B}$ is much larger than the conductivity in the directions perpendicular to $\boldsymbol{B}$; as a matter of fact, the parallel conductivity in the E-region ionosphere is about two orders of magnitude larger than the perpendicular conductivity at $100 \mathrm{~km}$ in altitude, and three orders of magnitude larger at $120 \mathrm{~km}$ in altitude. This ensures that in steady state the electric field perpendicular to $\boldsymbol{B}$ will map essentially unattenuated through the E-region ionosphere, as long as the

Published by Copernicus Publications on behalf of the European Geosciences Union. 


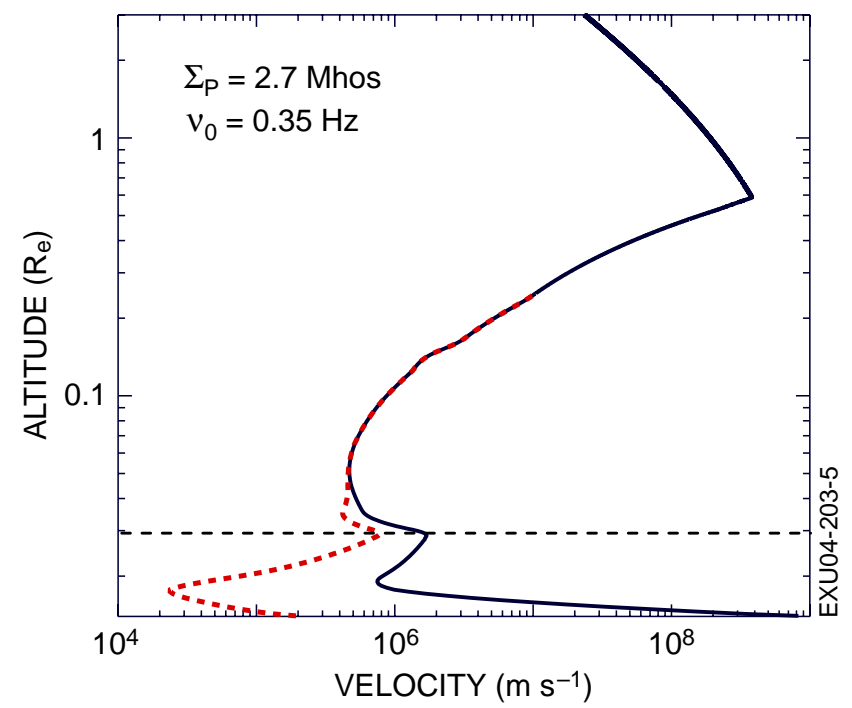

Fig. 1. Alfvén velocity (solid line) and phase velocity for Alfvén waves (red dashed line) in a representative ionosphere, and up to $3 R_{\mathrm{E}}$. The horizontal dashed line at $200 \mathrm{~km}$ marks the top of the E-region ionosphere, and the input to the "magnetospheric transmission line."

scale on which it varies in the direction perpendicular to $\boldsymbol{B}$ everywhere exceeds about one kilometer. However, in the transient case, there is a time constant associated with the penetration of an electric field through the ionosphere.

Electric field perturbations are transmitted along magnetic field lines by Alfvén waves (Mallinckrodt and Carlson, 1978). Figure 1 shows the phase velocity for Alfvén waves (red dashed line) along with the traditional (ion-neutral collisions not included) Alfvén velocity (solid line) in a representative ionosphere, and up to $3 R_{\mathrm{E}}$. In the E-region (below about $200 \mathrm{~km}$ ) collisions cause the Alfvén wave phase velocity to slow down dramatically; at $100 \mathrm{~km}$ the phase velocity is only $20 \mathrm{~km} \mathrm{~s}^{-1}$. The assumption - made in previous treatments of the IFI - that electric fields perpendicular to $\boldsymbol{B}$ map through the ionosphere, so that the ionosphere can be described by its field line integrated Pedersen and Hall conductivities, applies only in the case where the temporal scale for variations is much longer than the time for an Alfvén wave to propagate through the ionosphere. For the phase velocity of $20 \mathrm{~km} \mathrm{~s}^{-1}$, this means that the temporal scale for variations should be much longer than a few seconds. This condition is not met for the IFI, which involves the fundamental resonance of the IAR, with a frequency of about $0.5 \mathrm{~Hz}$.

The growing waves of the IFI have wavelengths of a few kilometers, and propagate relative to the ionospheric plasma with velocities of a few kilometers per second. If the time constant for electric field penetration begins to approach one wave period, in the reference frame fixed to the E-region plasma, then the penetration time constant will effect the wave. We show below that this effect of ionospheric thick-
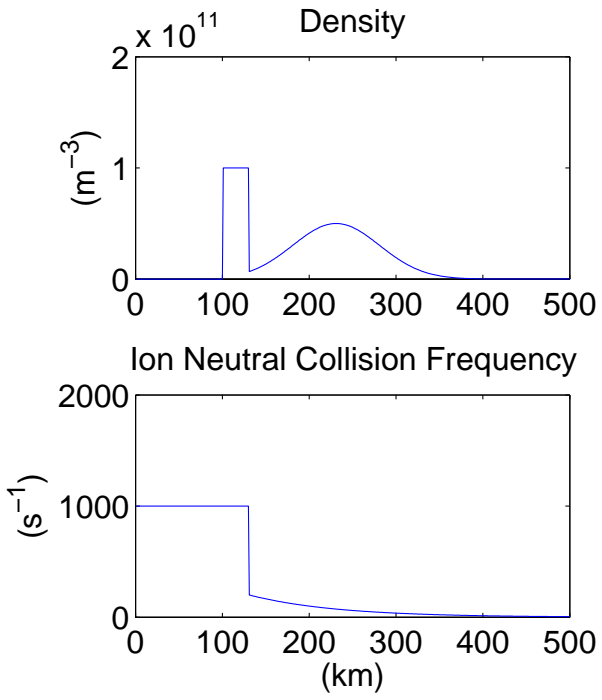

Fig. 2. Schematic of the physical model. The eigenmodes for the model will be found analytically.

ness leads to cutting off of the fundamental "thin-layer" mode, relevant higher order modes, and an associated orderof-magnitude reduction of the IFI growth rate.

\section{Growth rate derivation with E-region depth}

Figure 2 illustrates the ionospheric part of the model that will be solved. Moving down from $500 \mathrm{~km}$ in altitude the ion neutral collision frequency follows a realistic curve determined from the MSIS model. The plasma density also follows a realistic F-region curve taken from an incoherent scatter radar measurement. At a chosen altitude (shown as $130 \mathrm{~km}$ ) the collision frequency transitions abruptly into an E-region type value, and the plasma density transitions abruptly into a value representative of an auroral arc. These E-region parameters stay constant over some chosen arc thickness (shown as $30 \mathrm{~km}$ ), below which the plasma density abruptly decreases to zero.

The parameter values just above the E-region to F-region transition are representative of an ionospheric altitude of $200 \mathrm{~km}$, that is, a region of the ionosphere has been excised (70 km thick, for the case of Fig. 2), and all the F-region parameters shown in Fig. 2 are actually representative of a somewhat higher altitude than indicated (70 km higher). This is done in order to produce a model that is readily soluble using analytical techniques. The relatively low collision frequency above $200 \mathrm{~km}$ ensures that modifications of the plasma density produce negligibly small modifications of the conductivity, as compared to similar density modulations in the E-region. This means that to a good approximation, only the $30 \mathrm{~km}$ thick E-region need be considered as modifiable (active); the F-region, and on up into the magnetosphere, can be described simply as a medium through which 
Alfvén waves propagate. The region above $130 \mathrm{~km}$ as shown in Fig. 2 is equivalent to the Alfvén phase velocity profile above $200 \mathrm{~km}$ as shown in Fig. 1. Figure 1 shows how the model is continued on up to $20000 \mathrm{~km}$, above which it is assumed that the Alfvén velocity remains uniform.

Fluid equations will be used to describe the active E-region dynamics in two dimensions (one along $\boldsymbol{B}$, and one perpendicular to $\boldsymbol{B}$ ). In solving the fluid equations, the region below the arc (below $100 \mathrm{~km}$, as shown in the figure) will initially be taken to have a small non-zero density, but this value will be allowed to approach zero before a final result is obtained. Recombination will be omitted, and the fluid temperature will be set to zero. These last two simplifications will produce an overestimation of the instability growth rate. However, we do not expect them to produce a fundamental modification of the system behavior.

In summary, the excision of the altitude range between $200 \mathrm{~km}$ and the top of the arc, and the sharpening of the arc boundaries into a rectangular profile, constitute the major simplifications that must be made to produce a model that can be readily solved using analytical techniques. These simplifications are, at any rate, less draconian than taking the ionosphere to be infinitely thin. The model is representative of the ionosphere magnetosphere system with an active Eregion of finite thickness. Additional discussion of this point is given in Sect. 4.

\subsection{Treatment of the magnetosphere}

Because we are mainly interested in assessing the effects of ionospheric thickness, and because the magnetosphere is the passive element in the ionospheric feedback instability, we will treat the magnetosphere simply as a complex admittance $Y_{M}$ (following Sato, 1978). This provides for an accurate treatment of the shear Alfvén wave mode, using actual radar data to describe the F-region ionosphere, but does not account for mode conversion (Pokhotelov et al., 2001). A polarization electric field generated in the ionosphere launches an Alfvén wave propagating into the magnetosphere (Maltsev et al., 1977). Hence, if the magnetosphere is uniform, the ionosphere can be viewed as loaded by a transmission line with the Alfvén admittance $Y_{\mathrm{A}}$. If the properties of the magnetosphere vary with distance from the ionosphere, then waves initiated in the ionosphere will undergo partial reflection as they travel. According to the theory of transmission line networks, the effect of these partial reflections is to present the ionosphere with a modified admittance $Y_{\mathrm{M}}$. We now describe the calculation of this modified admittance for a realistic magnetosphere, which will also include the Fregion ionosphere; since the F-region does not play an active role in the IFI, it can be lumped into the passive load that is presented to the top of the E-region ionosphere.

Because the displacement current is negligible, an Alfvén wave is associated with a current $\boldsymbol{j}=\boldsymbol{\nabla} \times \boldsymbol{B} / \mu_{0}$. Assuming the form
$\boldsymbol{E} \propto \mathrm{e}^{i(\omega t-\boldsymbol{k} \cdot \boldsymbol{x})}$

for the Alfvén wave electric field, and using $\boldsymbol{\nabla} \times \boldsymbol{E}=-\frac{\partial \boldsymbol{B}}{\partial t}$, gives $\boldsymbol{B}=\boldsymbol{k} \times \boldsymbol{E} / \omega$. Combining these results gives the relationship between the current and electric field for an Alfvén wave:

$\boldsymbol{j}=\boldsymbol{\nabla} \times(\boldsymbol{k} \times \boldsymbol{E}) /\left(\mu_{o} \omega\right)=[\boldsymbol{k} \nabla \cdot \boldsymbol{E}-(\boldsymbol{k} \cdot \nabla) \boldsymbol{E}] /\left(\mu_{0} \omega\right)$.

If we assume $\boldsymbol{E} \cdot \hat{B}_{0}=0$ (where $\boldsymbol{B}_{0}=B_{0} \hat{B}_{0}$ is the geomagnetic field), and take $\boldsymbol{E}$ as the electric field at the input to the "magnetospheric transmission line," then the launching of an Alfvén wave upward into the magnetosphere is associated with a field aligned current

$$
\begin{aligned}
\boldsymbol{j} \cdot \hat{\boldsymbol{B}}_{0} & =\frac{\boldsymbol{k} \cdot \hat{\boldsymbol{B}}_{0}}{\mu_{0} \omega} \nabla_{\perp} \cdot \boldsymbol{E}, \text { orspecifically, } \\
j_{z} & =Y_{A} \boldsymbol{\nabla}_{\perp} \cdot \boldsymbol{E}, \text { where, } \\
Y_{A} & =\left(\left|\operatorname{real}\left(\boldsymbol{k} \cdot \hat{B}_{0}\right)\right|-i\left|\operatorname{imag}\left(\boldsymbol{k} \cdot \hat{B}_{0}\right)\right|\right) /\left(\mu_{0} \omega\right),
\end{aligned}
$$

and where $\hat{z}=-\hat{B}_{0}$ in the Northern Hemisphere, $\hat{z}=\hat{B}_{0}$ in the Southern Hemisphere, and $\nabla_{\perp}$ denotes the gradient in the plane perpendicular to $\boldsymbol{B}_{0}$. (Equation (3) assumes the convention (1).) We will refer to $Y_{\mathrm{A}}$ as the Alfvén admittance. This field aligned current must be drawn out of the ionosphere, and continuity of this current is the boundary condition imposed by the magnetosphere, in the case where the magnetosphere is uniform.

In the real case, where the magnetosphere is not uniform, the wave launched upward will undergo partial reflection as it travels, and hence there will also be a reflected wave incident on the ionosphere. The effect of this superposition of waves on the relationship between $\boldsymbol{j}$ and $\boldsymbol{E}$ at the input to the transmission line, that is, on the input admittance $Y_{\mathrm{M}}$, where

$j_{z}=Y_{\mathrm{M}} \nabla_{\perp} \cdot \boldsymbol{E}$,

is the purview of transmission line theory. To compute $Y_{\mathrm{M}}$ we appeal to the following fundamental result of transmission line theory: the driving-point admittance $Y_{\mathrm{S}}$ of a transmission line of characteristic admittance $Y_{0}$, length $l$, and propagation constant $\gamma$, loaded at the output by admittance $Y_{\mathrm{L}}$, is given by (e.g., Collin, 1966)

$Y_{S}=Y_{0} \frac{Y_{0} \sinh (i \gamma l)+Y_{L} \cosh (i \gamma l)}{Y_{0} \cosh (i \gamma l)+Y_{L} \sinh (i \gamma l)}$.

We apply Eq. (5) by assuming a horizontally stratified magnetosphere, divided into 10000 slabs of thickness $2 \mathrm{~km}$ each. Within each slab the transmission line parameters are given by the local Alfvén parameters. The upper section $(N$-th section) is terminated by a matched load. Therefore, from Eq. (5), the driving-point admittance $Y_{S N}$ seen looking into the $N$-th section is computed by taking $Y_{0 N}$ as the load $\left(Y_{L N}=Y_{0 N}\right.$, which gives $\left.Y_{S N}=Y_{0 N}\right)$, where $Y_{0 N}$ is the characteristic admittance of the $N$-th section; the drivingpoint admittance $Y_{S(N-1)}$ seen looking into the $(N-1)$-th 


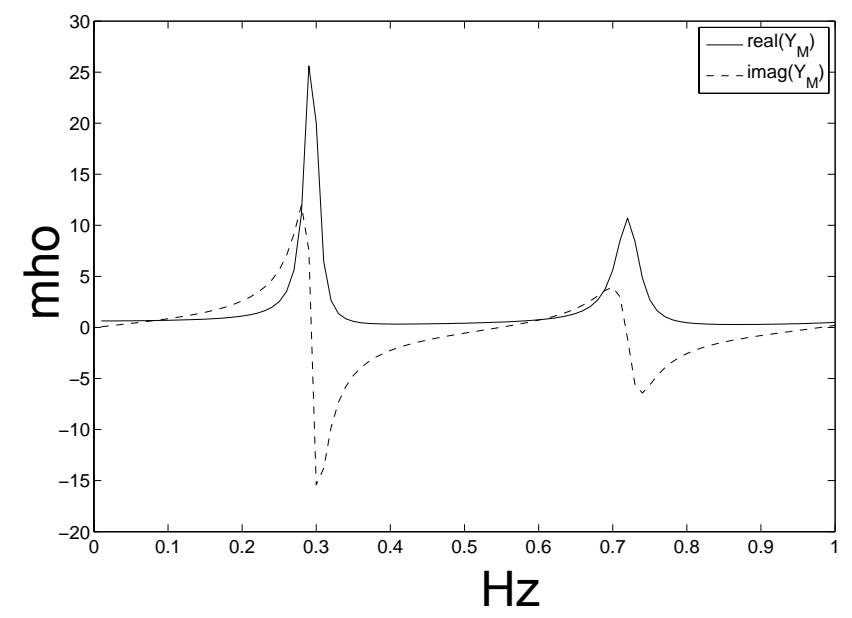

Fig. 3. Magnetospheric admittance computed from the transmission line model, using the parameters of Fig. 1.

section is computed by taking $Y_{S N}$ as the load $\left(Y_{L(N-1)}=\right.$ $\left.Y_{S N}\right)$; the driving-point admittance $Y_{S(N-2)}$ seen looking into the $(N-2)$-th section is computed by taking $Y_{S(N-1)}$ as the load $\left(Y_{L(N-2)}=Y_{S(N-1)}\right)$, and etc, until $Y_{S 1}$ is obtained. The magnetospheric admittance for use in Eq. (4) is then $Y_{\mathrm{M}}=Y_{S 1}$.

Because the Alfvén wave energy travels along magnetic field lines, and because of the assumption of a horizontally stratified magnetosphere, the distance between reflection points should be measured along magnetic field lines, and the relevant propagation constant should be taken parallel to the magnetic field lines. With this understanding, the characteristic admittance $Y_{0}$ of each slab is the local Alfvén admittance $\left(Y_{0}=Y_{\mathrm{A}}\right)$, and the propagation constant is derived from the parallel component of the wavevector (specifically, $\gamma=\boldsymbol{k} \cdot \hat{B}_{0}$ ). The wavevector is found from the dispersion relation for shear Alfvén waves (see derivation in Appendix B),

$k^{2}=\frac{2 \omega\left(\omega-i v_{\text {in }}\right)}{V_{\mathrm{A}}^{2}\left(1+\cos ^{2} \theta-\sqrt{\sin ^{4} \theta+4 \cos ^{2} \theta\left(\omega-i v_{\text {in }}\right)^{2} / \Omega_{i}^{2}}\right)},(6)$

where $\theta$ is the angle between $\boldsymbol{k}$ and $\boldsymbol{B}_{0}, k=|\boldsymbol{k}|, v_{\text {in }}$ is the ion-neutral collision frequency, $\Omega_{i}$ is the ion gyrofrequency, $V_{\mathrm{A}}=B_{0} / \sqrt{\mu_{0} m_{i} n}$ is the Alfvén velocity, $m_{i}$ is the ion mass, $n$ is the plasma density, and $\mu_{0}$ is the free space permeability. The collisions have been included in the dispersion relation so that the effect of the ionospheric F-region conductivity can be included in $Y_{\mathrm{M}}$; the input to the magnetospheric transmission line will be at the top of the E-region ionosphere. For our application $\theta \cong 90^{\circ}$, and $\left|\omega-i v_{\text {in }}\right|^{2} \ll \Omega_{i}^{2}$, so that (6) simplifies to

$\boldsymbol{k} \cdot \hat{B}_{0}=k \cos \theta= \pm \sqrt{\omega\left(\omega-i \nu_{\text {in }}\right)} / V_{\mathrm{A}}$,

which fills out the relations necessary to compute $Y_{\mathrm{M}}$ for a given $\omega$, profile of $v_{\text {in }}$, and profile $\boldsymbol{B}_{0}$.
This procedure has been applied using model parameters along with a selected, ISR measured F-region conductivity profile. The parameters used are summarized in Fig. 1, by plotting the profile for Alfvén velocity $V_{\mathrm{A}}$, and the profile for the parallel phase velocity $V_{\mathrm{p}}=\omega / \operatorname{real}\left(\boldsymbol{k} \cdot \hat{B}_{0}\right)=$ $V_{\mathrm{A}} / \operatorname{real}\left(\sqrt{1-i v_{\text {in }} / \omega}\right)$ (from Eq. 7), for $\omega=2 \pi(0.35 \mathrm{~Hz})$. The horizontal dashed line at $200 \mathrm{~km}$ marks the top of the E-region ionosphere, and the input to the "magnetospheric transmission line." Note that there is a region above the reference line where ion-neutral collisions are important. However, omitting collisions entirely from Eq. (7) has no significant effect on the calculations presented in this paper, and no effect at all on the conclusions. The relevance of $v_{\text {in }}$ in Eq. (7) lies only in the discussion of the introductory section (Sect. 1), where we noted that the phase velocity for Alfvén waves in the E-region ionosphere is much less than $V_{\mathrm{A}}$.

The resulting magnetospheric admittance $Y_{\mathrm{M}}$ looking up from the top of the E-region, which will be used to define the magnetospheric boundary condition according to Eq. (4), is plotted versus frequency $(\omega / 2 \pi)$ in Fig. 3. Note the resonances (where the imaginary part of the admittance vanishes) that occur at about $0.3 \mathrm{~Hz}$ and $0.7 \mathrm{~Hz}$. These are analogous to the resonance of an electric circuit, when the inductive part cancels the capacitive part. By placing a resistor across the input, to model a thin E-region load, the circuit becomes a model of the ionospheric Alfvén resonator; the zero of the imaginary part of the admittance determines the resonant frequency, and the resistor value determines the quality factor. A better model for a passive ionosphere could be obtained by continuing the transmission line down to an altitude below where the ionospheric conductivity vanishes, with the input left as an open circuit, in which case the electrical length of the E-region is included. However, in this work we will apply a model for an active E-region, with electrical length, and find unstable resonances.

\subsection{General dispersion relation}

Derivation of the IFI growth rate requires consideration of the ionospheric depth. We now solve for the waves in a finite thickness ionosphere that satisfy the magnetospheric boundary condition (Eq. 4), which means that a dependence will be included in the direction of the magnetic field.

Alfvén waves are electromagnetic waves, in general. However, the Alfvén wavevector $\boldsymbol{k}$ may make any angle $\theta$ with respect to the background magnetic field $\boldsymbol{B}_{0}$, which places $\boldsymbol{k}$ at an angle $\theta+\pi / 2$ with the electric field $\boldsymbol{E}$. In the limit as $\theta \rightarrow \pi / 2, \boldsymbol{k}$ becomes aligned with the electric field $\boldsymbol{E}$, so that $\nabla \times \boldsymbol{E}=\boldsymbol{k} \times \boldsymbol{E} \rightarrow 0$; the Alfvén wave becomes electrostatic. In this work we are considering perpendicular to $\boldsymbol{B}_{0}$ wavelengths on the order of $1 \mathrm{~km}$, whereas the wavelength along $\boldsymbol{B}_{0}$ at the top of the E-region (200 km altitude) is about $6 \times 10^{5} \mathrm{~km}$ (from Fig. 1, with a characteristic frequency of $1 \mathrm{~Hz}$ ). This gives $\theta=\pi / 2-0.000002$, so that the Alfvén 
wave at the top of the E-region is very nearly electrostatic. We will likewise solve for the response in the E-region ionosphere using the electrostatic assumption. This assumption is usually employed in the ionosphere, even for much higher frequency waves involving parallel electric fields (e.g., Ossakow et al., 1975; Janhunen, 1997). A thorough justification for the case of Rayleigh-Taylor waves in the F-region can be found in Basu (2005). For completeness, in the appendix we demonstrate the validity of the electrostatic assumption for our particular problem, and derive the working equations given below from the full set of fluid equations together with Maxwell's equations.

Consider auroral plasma in the altitude range where the electrons are magnetized, and the ions are collisional, specifically, the altitude range where $\kappa_{i}=\frac{\Omega_{i}}{v_{\text {in }}} \ll 1, \kappa_{e}=\frac{\Omega_{e}}{v_{e n}} \gg 1$, and $\kappa_{i} \gg \frac{1}{\kappa_{e}}$, where $\Omega_{i}$ is the ion gyro frequency, $\Omega_{e}$ is the electron gyro frequency, $\nu_{\text {in }}$ is the ion-neutral collision frequency, and $v_{e n}$ is the electron neutral collision frequency. These approximations are valid roughly in the altitude range from $100 \mathrm{~km}$ to $120 \mathrm{~km}$. Choose an orthogonal coordinate system with $\hat{z}$ directed upward (and assumed opposite to the magnetic field), and $\hat{x} \times \hat{y}=\hat{z}$. With these approximations the zero temperature ionospheric fluid equations for the steady state ion velocity $\boldsymbol{v}_{i}$, and the $x$ and $z$ components of the current density, $j_{x}$ and $j_{z}$, simplify as follows:

$$
\begin{aligned}
\boldsymbol{v}_{i} & =\frac{-\kappa_{i}}{B}\left(\boldsymbol{\nabla} \phi-\boldsymbol{E}_{0}-\boldsymbol{u} \times \boldsymbol{B}_{0}\right)+\boldsymbol{u} \\
j_{x} & =n e \frac{-\kappa_{i}}{B}\left(\frac{\partial \phi}{\partial x}-E_{0 x}+u_{y} B_{0}\right)+n e \frac{1}{B_{0}}\left(E_{y 0}+u_{x} B_{0}\right) \\
j_{z} & =n e \frac{-\kappa_{e}}{B} \frac{\partial \phi}{\partial z} \\
0 & =\frac{\partial j_{x}}{\partial x}+\frac{\partial j_{z}}{\partial z} \\
0 & =\frac{\partial n}{\partial t}+\frac{\partial}{\partial x}\left(n v_{i x}\right)+\frac{\partial}{\partial z}\left(n v_{i z}\right)
\end{aligned}
$$

where $\boldsymbol{E}_{0}$ (and its components $E_{0 x}, E_{0 y}$, and $E_{0 z}$ ) represents a uniform and constant background electric field, $\boldsymbol{u}$ (and its components $u_{x}$, and $u_{y}$ ) represents a uniform constant wind velocity, $n$ is the plasma density, $\boldsymbol{B}_{0}=-B_{0} \hat{z}$ is the background geomagnetic field, and $\phi$ is an electrostatic potential such that the total electric field is $\boldsymbol{E}=\boldsymbol{E}_{0}-\nabla \phi$. We have assumed that the background electric field is perpendicular to $\boldsymbol{B}_{0}$, i.e., that $E_{0 z}=0$. Equations (11) and (12) are the current and ion continuity equations, respectively, under the assumption that there is no variation in the $y$ direction; we will use this assumption throughout.

The equation for the magnetospheric boundary condition (4) applies in the frame of reference moving with the $\boldsymbol{E} \times \boldsymbol{B}_{0}$ drift, which is the frame of reference with $E_{0 x}=$ $E_{0 y}=0$. Therefore, we will work in this frame of reference, so that $\boldsymbol{E}_{0}=0$ throughout.
Expressing $n v_{i x}$ and $n v_{i z}$ in terms of $j_{x}$ and $j_{z}$, substituting the result into Eq. (12), using Eq. (11) to eliminate $j_{x}$, and finally using Eq. (10) to express $j_{z}$ in terms of $\phi$, gives

$$
\frac{\partial n}{\partial t}=\frac{-1}{B_{0}}\left(\kappa_{e}-\kappa_{i}\right)\left[\frac{\partial n}{\partial z} \frac{\partial \phi}{\partial z}+n \frac{\partial^{2} \phi}{\partial z^{2}}\right] .
$$

Substituting Eqs. (9) and (10) into Eq. (11) gives

$$
\begin{aligned}
\frac{e \kappa_{e}}{B} \frac{\partial n}{\partial z} \frac{\partial \phi}{\partial z}+\frac{e \kappa_{e}}{B} n \frac{\partial^{2} \phi}{\partial z^{2}}= & \frac{e \kappa_{i}}{B} \frac{\partial n}{\partial x}\left(-u_{y} B-\frac{\partial \phi}{\partial x}\right) \\
& -\frac{e \kappa_{i}}{B} n \frac{\partial^{2} \phi}{\partial x^{2}}+e u_{x} \frac{\partial n}{\partial x} .
\end{aligned}
$$

Finally, substituting Eq. (10) into the magnetospheric boundary condition (4) gives

$$
\left.\frac{\partial^{2} \phi}{\partial x^{2}}\right|_{z_{T}}=\left.n \frac{e \kappa_{e}}{B_{0} Y_{M}} \frac{\partial \phi}{\partial z}\right|_{z_{T}},
$$

where the subscript indicates that Eq. (15) applies only at $z=$ $z_{T}$, which we take to be the top of the E-region ionosphere.

Equations (13) through (15) will be solved in linearized form: specifically, with $n=n_{0}+\delta n$, where $n_{0}=n_{0}(z)$ is a time independent background density profile depending only on $z$ (specified below), and discarding terms that are second order or higher in $\delta n$ and $\phi$. In addition, $\delta n$ and $\phi$ will be assumed to have the form

$$
\begin{aligned}
\delta n & =N(z) \mathrm{e}^{i(\omega t-k x)} \\
\phi & =\Phi(z) \mathrm{e}^{i(\omega t-k x)},
\end{aligned}
$$

so that they are wavelike in the x-direction. The arbitrary functions $N(z)$ and $\Phi(z)$ will be determined uniquely by the requirement that Eq. (16) satisfy Eqs. (13) through (15) for $z \leq z_{T}$, with the boundary condition that $\delta n$ is bounded as $z \rightarrow-\infty$, and for the chosen function $n_{0}=n_{0}(z)$. The resulting solutions for $\delta n$ and $\phi$ constitute natural modes of the system, which are unstable if imag $(\omega)<0$.

In order to represent the effect of the finite thickness (not zero, and not infinite) of the ionosphere in the simplest possible way, we choose a slab model

$n_{0}(z)=n_{01} \mathrm{H}(z)+n_{02}$,

where $\mathrm{H}(z)$ is the Heaviside step function, so that the ionosphere has density $n_{0}=n_{01}+n_{02}$ in the region $0<z \leq z_{T}$, and density $n_{0}=n_{02}$ for $z<0$. The magnetospheric boundary condition (15) is applied at $z=z_{T}$. The derivative of $\mathrm{H}(z)$ is the Dirac delta function, $\frac{\mathrm{dH}}{\mathrm{d} z}=\delta(z)$. The density at $z=0$ is found from the consistency of this definition:

$$
\begin{aligned}
\mathrm{H}(0) & =\int_{-B}^{A} \mathrm{~d} z \delta(z) \mathrm{H}(z) \\
& =\int_{-B}^{A} \mathrm{~d} z\left[\frac{\mathrm{d}}{\mathrm{d} z}\left(\mathrm{H}^{2}(z)\right)-\mathrm{H}(z) \frac{\mathrm{d}}{\mathrm{d} z} \mathrm{H}(z)\right]
\end{aligned}
$$




$$
\begin{aligned}
& =\left.\mathrm{H}^{2}(z)\right|_{-B} ^{A}-\int_{-B}^{A} \mathrm{~d} z \mathrm{H}(z) \delta(z)=1-\mathrm{H}(0) \\
& \rightarrow \mathrm{H}(0)=\frac{1}{2}
\end{aligned}
$$

so that $n_{0}(0)=n_{01} / 2+n_{02}$.

Substituting Eq. (16) into Eqs. (13) and (14), discarding terms that are second order or higher in $\delta n$ and $\phi$, eliminating $N(z)$ between the two equations, and using the zeroth order density profile (17) along with the various delta function derivative relations, gives

$$
\begin{gathered}
\frac{\kappa_{i}}{\kappa_{e}}\left(n_{01} \mathrm{H}(z)+n_{02}\right) \omega k \Phi+\left(u_{x}-\kappa_{i} u_{y}-\omega / k\right) n_{01} \delta(z) \frac{\mathrm{d} \Phi}{\mathrm{d} z} \\
+\left(u_{x}-\kappa_{i} u_{y}-\omega / k\right)\left(n_{01} \mathrm{H}(z)+n_{02}\right) \frac{\mathrm{d}^{2} \Phi}{\mathrm{d} z^{2}}=0
\end{gathered}
$$

Similarly, the boundary condition (15) becomes

$$
-\left.k^{2} \Phi\right|_{z_{T}}=\left.n_{0} \frac{e \kappa_{e}}{B_{0} Y_{M}} \frac{\partial \Phi}{\partial z}\right|_{z_{T}} .
$$

Away from $z=0$, Eq. (18) reduces to

$$
\frac{\kappa_{i}}{\kappa_{e}} \omega k \Phi+\left(u_{x}-\kappa_{i} u_{y}-\omega / k\right) \frac{\mathrm{d}^{2} \Phi}{\mathrm{d} z^{2}}=0,
$$

which is an ordinary differential equation with constant coefficients, with solutions of the form $\mathrm{e}^{m z}$. Substituting this form gives

$$
m=\sqrt{k^{2} \frac{\kappa_{i}}{\kappa_{e}} \frac{\omega}{\omega-k\left(u_{x}-\kappa_{i} u_{y}\right)}}
$$

We look for a solution to Eq. (18) in the form

$\Phi(z)=\left(c_{1} \mathrm{H}(z)+c\right) \mathrm{e}^{\sigma z}+\left(c_{2} \mathrm{H}(z)+c_{3}\right) \mathrm{e}^{-\sigma z}$,

where $\sigma=\operatorname{sign}(\operatorname{Re}(m)) m$,

where $\operatorname{Re}(m)$ denotes the real part of $m$. Applying the boundary condition $\Phi \rightarrow 0$ as $z \rightarrow-\infty$, gives $c_{3}=0$. Making $\frac{\partial \Phi}{\partial z}$ (the field-aligned electric field) finite at $z=0$ requires $c_{1}=-c_{2}$. With these stipulations, substituting Eq. (22) into Eq. (18), and integrating over a small region around $z=0$, gives $c=\left(4 \mathrm{H}(0)+2 n_{02} / n_{01}\right) c_{2}=2\left(1+n_{02} / n_{01}\right) c_{2}$. This determines the solution for $\Phi$, and using Eq. (13) or (14), also the solution for $N$ :

$$
\begin{aligned}
\Phi(z)= & c_{2}\left[\left(2+2 \frac{n_{02}}{n_{01}}-\mathrm{H}(z)\right) \mathrm{e}^{\sigma z}+\mathrm{H}(z) \mathrm{e}^{-\sigma z}\right] \\
N(z)= & \frac{i c_{2}}{k B_{0}\left(u_{x}-\kappa_{i} u_{y}\right)}\left(\kappa_{i} k^{2}-\kappa_{e} \sigma^{2}\right)\left(n_{01} \mathrm{H}(z)+n_{02}\right) \\
& {\left[\mathrm{H}(z)\left(\mathrm{e}^{\sigma z}-\mathrm{e}^{-\sigma z}\right)-2\left(1+\frac{n_{02}}{n_{01}}\right) \mathrm{e}^{\sigma z}\right] }
\end{aligned}
$$

It remains to find the dispersion relation between $\omega$ and $k$, which is determined by the boundary condition (19). Substituting Eq. (23) into Eq. (19) gives

$\sigma=-k^{2} \frac{B_{0} Y_{M}}{e \kappa_{e}\left(n_{01}+n_{02}\right)} \frac{\left(1+2 n_{02} / n_{01}\right) \mathrm{e}^{\sigma z_{T}}+\mathrm{e}^{-\sigma z_{T}}}{\left(1+2 n_{02} / n_{01}\right) \mathrm{e}^{\sigma z_{T}}-\mathrm{e}^{-\sigma z_{T}}}$.
Combining Eqs. (21) and (24) gives a transcendental equation for $\omega$ in terms of $k$, i.e., the dispersion relation (note, $\sigma=\operatorname{sign}(\operatorname{Re}(m)) m)$.

\subsubsection{Reduction when $n_{02} \rightarrow 0$, and for a thin ionosphere}

In the limit $z_{T} \rightarrow 0$ and $n_{02} \rightarrow 0$ the dispersion relation just derived should reduce to the expression derived by Sato (1978) involving field line integrated ionospheric conductivities. Applying the limit $n_{02} \rightarrow 0$ to Eq. (24) gives

$\sigma=-k^{2} \frac{B_{0} Y_{M}}{e \kappa_{e} n_{01} \tanh \left(\sigma z_{T}\right)}$.

Squaring Eq. (21) and using Eq. (25), after some manipulation, gives

$\omega=k \frac{\frac{u_{x} B_{0}}{\kappa_{i}}-u_{y} B_{0}}{\frac{B_{0}}{\kappa_{i}}+\frac{e n_{01} \tanh \left(\sigma z_{T}\right)}{\sigma Y_{M}}}$.

Equations (25) and (26) together form the transcendental dispersion relation in the limit $n_{02} \rightarrow 0$. Applying the limit $z_{T} \rightarrow 0$ to Eq. (26) gives

$\omega=k \frac{u_{x}-\kappa_{i} u_{y}}{1+\frac{e \kappa_{i} N}{Y_{M} B_{0}}}$,

where $N=n_{01} z_{T}$ is the field line integrated plasma density of the ionospheric layer. Equation (27) agrees with the expression derived by Sato (1978).

\subsubsection{Numerical solution of dispersion relation}

We will solve the dispersion relation numerically in the limit $n_{02} \rightarrow 0$, for $z_{T}$ finite. This means simultaneously solving Eqs. (25) and (26). Using various identities in hyperbolic trigonometry, it is possible to separate Eq. (25) into real and imaginary parts as

$$
\begin{aligned}
& \sigma_{r} \sinh \left(2 \sigma_{r} z_{T}\right)-\sigma_{i} \sin \left(2 \sigma_{i} z_{T}\right) \\
& =-k^{2} \frac{Y_{M r}}{\sigma_{0} n_{01}}\left(\cosh \left(2 \sigma_{r} z_{T}\right)+\cos \left(2 \sigma_{i} z_{T}\right)\right) \\
& i \sigma_{r} \sin \left(2 \sigma_{i} z_{T}\right)+i \sigma_{i} \sinh \left(2 \sigma_{r} z_{T}\right) \\
& =-k^{2} \frac{i Y_{M i}}{\sigma_{0} n_{01}}\left(\cosh \left(2 \sigma_{r} z_{T}\right)+\cos \left(2 \sigma_{i} z_{T}\right)\right),
\end{aligned}
$$

where $\sigma=\sigma_{r}+i \sigma_{i}$ and $Y_{\mathrm{M}}=Y_{M r}+i Y_{M i}$. Dividing the imaginary part by the real part eliminates $k$, and gives

$$
\left(\sigma_{r}+\sigma_{i} \frac{Y_{M i}}{Y_{M r}}\right) \sin \left(2 \sigma_{i} z_{T}\right)+\left(\sigma_{i}-\sigma_{r} \frac{Y_{M i}}{Y_{M r}}\right) \sinh \left(2 \sigma_{r} z_{T}\right)=0 .
$$

Equation (30) can be reliably solved using Newton's method.

Due to the oscillatory functions, the dispersion relation has many solutions, and it is necessary to sort through them and choose the ones of most interest. We will solve for the two solutions with the smallest $\sigma_{i}$, which we have found by example to be the two solutions with the largest growth rate. To 
Table 1. Fixed simulation parameters.

\begin{tabular}{ccccccc}
\hline Altitude & $n$ & $\kappa_{i}$ & $\kappa_{e}$ & $\Sigma_{P}$ & $\Sigma_{H}$ & $B$ \\
\hline $100 \mathrm{~km}$ & $10^{11} \mathrm{~m}^{-3}$ & $\frac{1}{56}$ & 83 & 0.29 mhos & $9.61 \mathrm{mhos}$ & $5 \times 10^{-5} \mathrm{~T}$ \\
$120 \mathrm{~km}$ & $10^{11} \mathrm{~m}^{-3}$ & $\frac{1}{4}$ & 1166 & 2.27 mhos & 9.05 mhos & $5 \times 10^{-5} \mathrm{~T}$ \\
\hline
\end{tabular}

do this we first choose a frequency $(\operatorname{Re}(\omega))$, and form a dense sampling of the real number line on an interval surrounding the the thin-layer value for $\sigma_{i}$ (obtained from Eqs. 25 and 27 with $z_{T} \rightarrow 0$ ). Each of these samples is substituted for $\sigma_{i}$ in Eq. (30), and Newton's method used to solve Eq. (30) for the associated $\sigma_{r}$. The result is a list of "candidate solutions" for $\sigma\left(=\sigma_{r}+i \sigma_{i}\right)$, with monotonically increasing $\sigma_{i}$. Finally, substitute this list into Eq. (29), and plot the left hand side minus the right hand side versus $\sigma_{i}$, that is, plot

$$
\begin{aligned}
\Theta\left(\sigma_{i}\right)= & \sigma_{r}\left(\sigma_{i}\right) \sin \left(2 \sigma_{i} z_{T}\right)+\sigma_{i} \sinh \left(2 \sigma_{r}\left(\sigma_{i}\right) z_{T}\right) \\
& +k^{2}\left(\sigma_{i}\right) \frac{Y_{M i}}{\sigma_{0} n_{01}}\left(\cosh \left(2 \sigma_{r}\left(\sigma_{i}\right) z_{T}\right)+\cos \left(2 \sigma_{i} z_{T}\right)\right)(31)
\end{aligned}
$$

where $\sigma_{r}\left(\sigma_{i}\right)$ is evaluated from the list of candidate solutions. In doing this, the constraint of Eq. (26) is enforced by evaluating $k\left(\sigma_{i}\right)$ as

$k\left(\sigma_{i}\right)=\operatorname{Re}(\omega) / \operatorname{Re}\left(\frac{\frac{u_{x} B_{0}}{\kappa_{i}}-u_{y} B_{0}}{\frac{B_{0}}{\kappa_{i}}+\frac{e n_{01} \tanh \left[\left(\sigma_{r}\left(\sigma_{i}\right)+i \sigma_{i}\right) z_{T}\right]}{\left(\sigma_{r}\left(\sigma_{i}\right)+i \sigma_{i}\right) Y_{M}}}\right)$,

where again $\sigma_{r}\left(\sigma_{i}\right)$ is evaluated from the list of candidate solutions. The zero crossings of $\Theta\left(\sigma_{i}\right)$ are the solutions to the dispersion relation; they are found by interpolation. The imaginary part of $\omega$ (the growth rate) is found from the imaginary part of Eq. (26).

Figure $4 \mathrm{a}-\mathrm{c}$ shows three examples of the function $\Theta\left(\sigma_{i}\right)$ that arise in the above method of solution, for three different layer thicknesses, with the density adjusted so that they all have the same field line integrated conductivity: Fig. $4 \mathrm{a}, 5 \mathrm{~km}$ thick; Fig. 4b, $10 \mathrm{~km}$ thick; and Fig. 4c, $15 \mathrm{~km}$ thick. The background electric field is $50 \mathrm{mV} / \mathrm{m}$; the altitude is $120 \mathrm{~km}$; the frequency analyzed is $0.5 \mathrm{~Hz}$; and the field line integrated conductivity (1.14 mhos) corresponds to a $15 \mathrm{~km}$ thick layer with an electron density of $10^{11} \mathrm{~m}^{-3}$. The vertical blue line marks $\sigma_{i}$ for the thin-layer solution. The vertical green (red) line marks $\sigma_{i}$ for the actual solution with the largest (second largest) growth rate.

Figure $4 \mathrm{a}-\mathrm{c}$ illustrates the fact that there are many solutions, or "modes" of the dispersion relation, and that the modes with the smallest $\sigma_{i}$ cutoff as the layer thickens. By numerical experimentation, we have found that the modes with the smallest $\sigma_{i}$ have the largest growth rate, at least for the three or four values of $\sigma_{i}$ that are closest to the smallest value. Therefore, the largest growth rate at any given frequency (among all the modes) is discontinuous with layer thickness.
Figure $4 \mathrm{c}$ shows that this discontinuity typically occurs at layer thicknesses that are thinner than the normal thickness for an auroral arc. In Sect. 3 we will see that the reduction in growth rate across the discontinuity is very substantial.

Figures $4 \mathrm{~d}-4 \mathrm{f}$ show the growth rates (of the fastest growing mode) as a function of frequency for the same parameters as Figs. $4 \mathrm{a}-4 \mathrm{c}$, respectively. For the thinnest layer thickness $(5 \mathrm{~km})$ the thin layer solution is reproduced except at the top of the frequency range. For the $10 \mathrm{~km}$ thick layer a discontinuity in the growth rate has appeared at about $0.85 \mathrm{~Hz}$, and the growth rate is also slightly reduced at proximate frequencies below $0.85 \mathrm{~Hz}$. This suggests that the small reduction in growth rate seen in Fig. 4d, at higher frequencies, can be traced to the presence of a discontinuity above the displayed frequency range. For the $15 \mathrm{~km}$ thick layer the discontinuity has moved below $0.5 \mathrm{~Hz}$, which is the frequency used in Figs. 4a-4c. Evidently, the discontinuity with frequency and the discontinuity with layer thickness are related. Thin layers will only behave as such up to some maximum frequency. This duality between the frequency and layer thickness response is consistent with the discussion given in the introduction.

\section{Results}

Figures 5 and 6 show the results plotted versus frequency as measured in the frame of reference moving with the $\boldsymbol{E}_{0} \times \boldsymbol{B}_{0}$ drift (i.e., the frame where $\boldsymbol{E}_{0}=0$ ), for $100 \mathrm{~km}$ (Fig. 5) and $120 \mathrm{~km}$ (Fig. 6) altitude layers (i.e., the collision frequency throughout the layer is set either to the value applicable at $100 \mathrm{~km}$, or to the value applicable at $120 \mathrm{~km}$ ). In both figures, panels (a) through (c) are the growth rate, wavelength, and frequency in the earth-fixed frame (determined by Doppler shift), respectively, when the background electric field in the earth fixed frame is $50 \mathrm{mV} \mathrm{m}^{-1}$. Panels (d) through (f) are the same when the background electric field is $100 \mathrm{mV} \mathrm{m}^{-1}$. In both cases the electric field is directed in the $\hat{y}$ direction (perpendicular to the wavevector). The integrated Pedersen conductivity $\left(\Sigma_{P}\right)$ for Fig. 5 is 0.29 mhos, which corresponds to a $30 \mathrm{~km}$ thick layer with an electron density of $10^{11} \mathrm{~m}^{-3}$, at $100 \mathrm{~km}$ in altitude. The integrated Pedersen conductivity for Fig. 6 is 2.27 mhos, which corresponds to the same except at $120 \mathrm{~km}$ in altitude. A list of additional simulation parameters is given in Table 1. The green (purple) lines show results for the mode with the largest (second largest) growth rate for a $30 \mathrm{~km}$ thick layer. The blue lines show results for an 

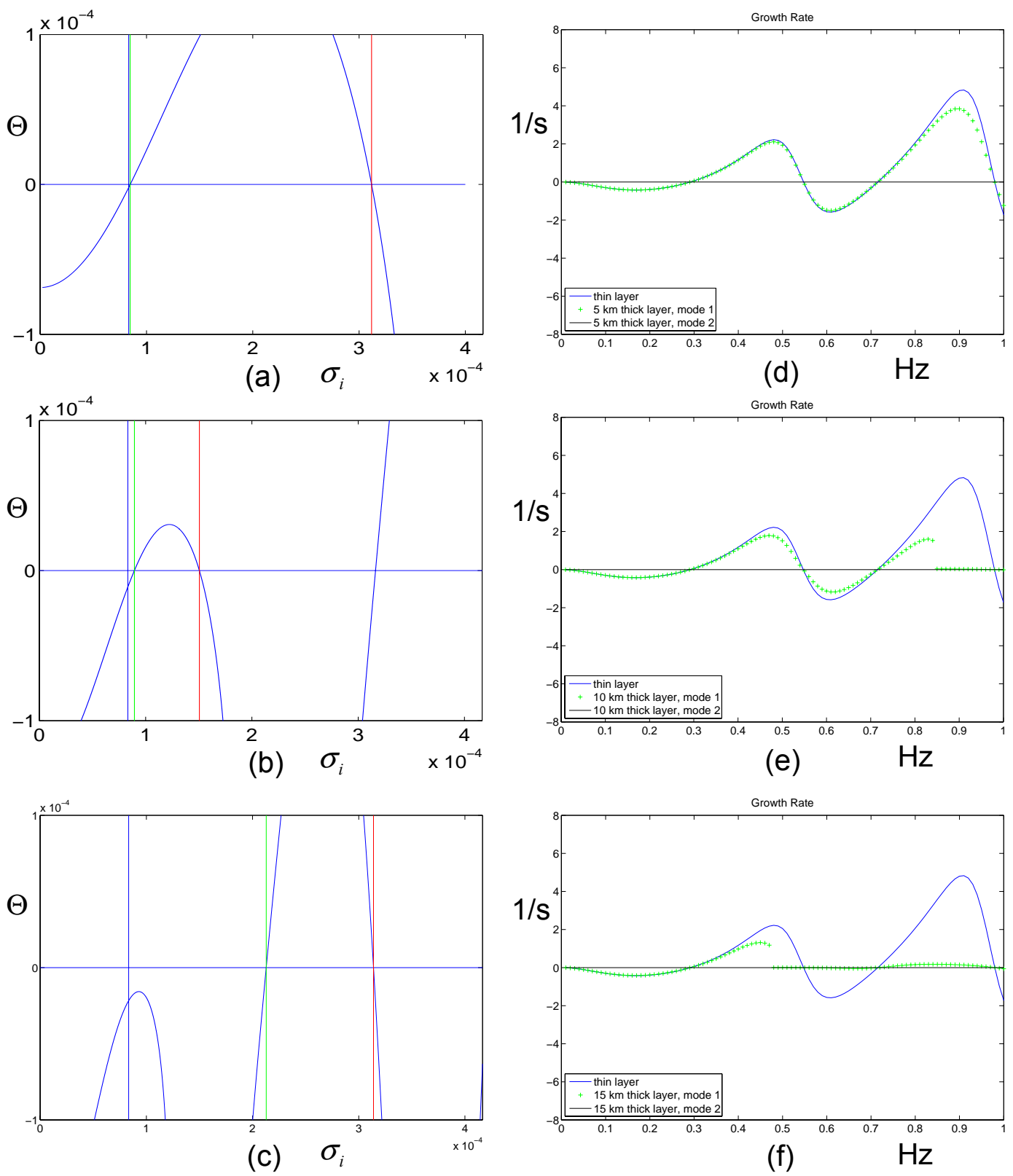

Fig. 4. Panels (a) through (c) show the function $\Theta\left(\sigma_{i}\right)$ plotted versus $\sigma_{i}$, for three different layer thicknesses all having the same integrated conductivity: (a) $5 \mathrm{~km}$ thick layer, (b) $10 \mathrm{~km}$ thick layer, and (c) $15 \mathrm{~km}$ thick layer. The vertical blue line marks $\sigma_{i}$ for the thin-layer solution. The vertical green (red) line marks $\sigma_{i}$ for the actual solution with the largest (second largest) growth rate. This shows the cutoff of the fundamental (thin-layer) mode when the layer becomes too thick. Panels (d) through (f) show the growth rate plotted versus frequency for the same three layer thicknesses: (d) $5 \mathrm{~km}$ thick, (e) $10 \mathrm{~km}$ thick, and (f) $15 \mathrm{~km}$ thick. These show the convergence of the growth rate to the thin layer value. For all, the background electric field is $50 \mathrm{mV} \mathrm{m}^{-1}$; the altitude is $120 \mathrm{~km}$; and the field line integrated Pedersen and Hall conductivities are 1.14 mhos and 4.53 mhos, respectively.

infinitely thin auroral layer of the same integrated conductivity, which we will refer to simply as a thin layer. The results for the frequency in the earth-fixed frame (black lines) are shown only for the mode with the largest growth rate when the layer is $30 \mathrm{~km}$ thick (i.e., for the green lines in the upper panels).
Figures 5 and 6 show that the growth rate is discontinuous in frequency, which, from the discussion of Sect. 2.2.2, is clearly a result of the cutting-off of the thin-layer solution (first eigenmode, with smallest $\sigma_{i}$ ). Figure 7 shows the density and potential eigenfunctions (from Eq. 23) below and above the cutoff frequency $(0.46 \mathrm{~Hz})$ of the thin-layer 


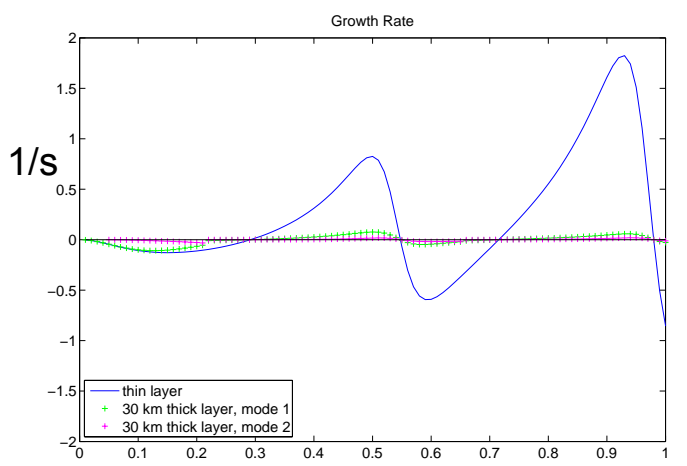

(a)

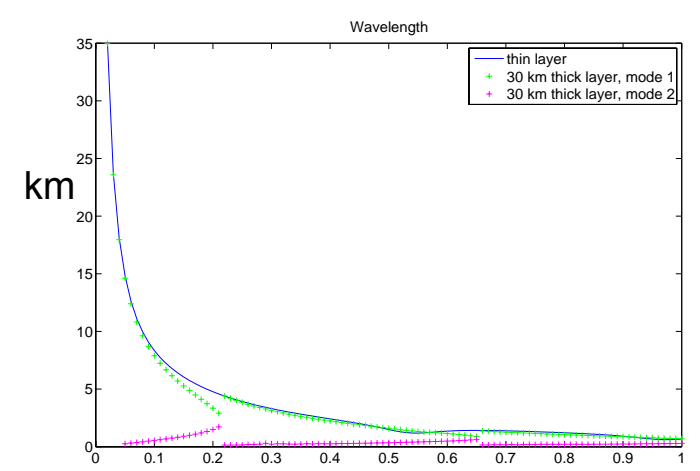

(b) $\mathrm{Hz}$

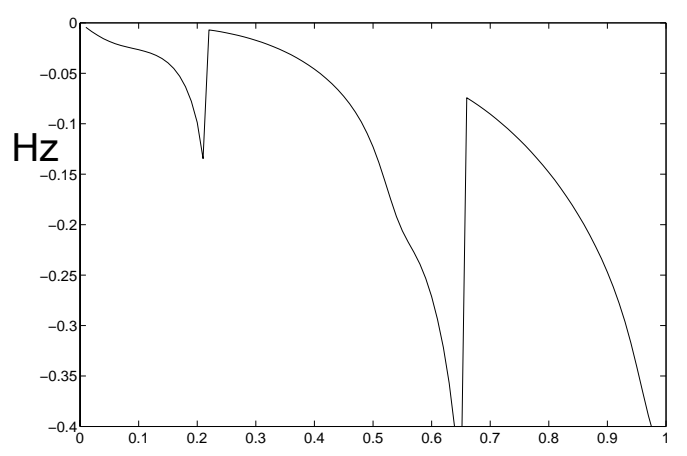

(c)

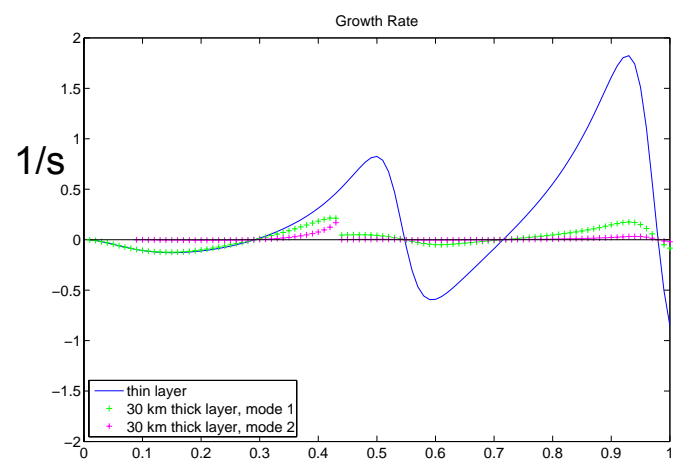

(d) $\quad \mathrm{Hz}$

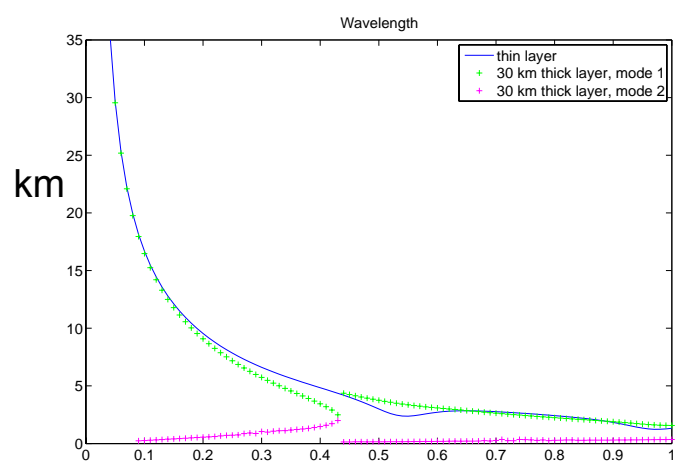

(e) $\mathrm{Hz}$

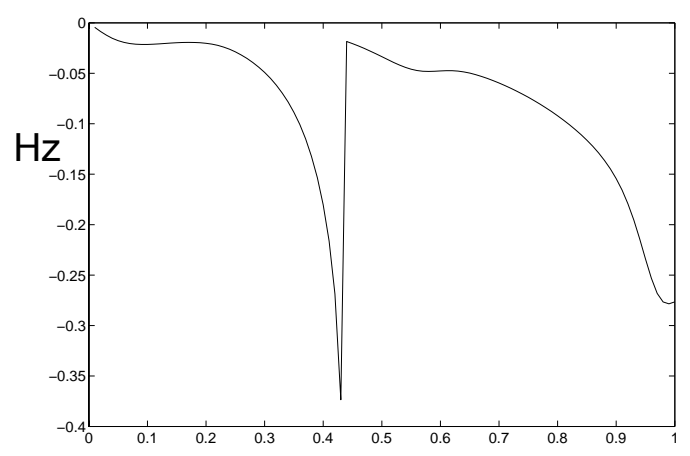

(f) $\quad \mathrm{Hz}$

Fig. 5. Growth rate, wavelength, and frequency in the earth-fixed frame, plotted versus frequency in the frame moving with the $\boldsymbol{E}_{0} \times \boldsymbol{B}$ velocity, for a $100 \mathrm{~km}$ altitude layer. Panels (a) through (c) are for $\boldsymbol{E}_{0}=50 \mathrm{mV} \mathrm{m}^{-1}$. Panels (d) through (f) are for $\boldsymbol{E}_{0}=100 \mathrm{mV} \mathrm{m}$. green (purple) lines show results for the mode with the largest (second largest) growth rate for a $30 \mathrm{~km}$ thick layer. The blue lines show results for a thin auroral layer of the same integrated conductivity. The results for the frequency in the earth-fixed frame (black lines) are shown only for the mode with the largest growth rate when the layer is $30 \mathrm{~km}$ thick (i.e., for the green lines). The integrated Pedersen and Hall conductivities are 0.29 mhos and 9.61 mhos, respectively, which corresponds to a $30 \mathrm{~km}$ thick layer with an electron density of $10^{11} \mathrm{~m}^{-3}$.

mode, for a $120 \mathrm{~km}$ altitude layer with $100 \mathrm{mV} / \mathrm{m}$ electric field. Panel (a) shows the percentage density perturbation for the mode with the maximum growth rate, just below cutoff of the thin layer mode. Panel (b) shows the associated potential function. Panel (c) shows the percentage density perturbation just above cutoff, for the mode with the highest growth rate, which is now the second eigenmode (the first eigenmode being cutoff). Panel (d) shows the associated potential function.
It is notable that the mode shown in panels (c) and (d) of Fig. 7 supports a parallel electric field, and cannot be represented by field line integrated quantities in any approximation. Figures $6 \mathrm{e}$ and $6 \mathrm{f}$ show that just before cutoff the frequency in the earth fixed frame increases rapidly, and the wavelength decreases, which supports the assertion that cutoff occurs because the electric field cannot map through the layer (along $\boldsymbol{B}_{0}$ ) with sufficient rapidity. 


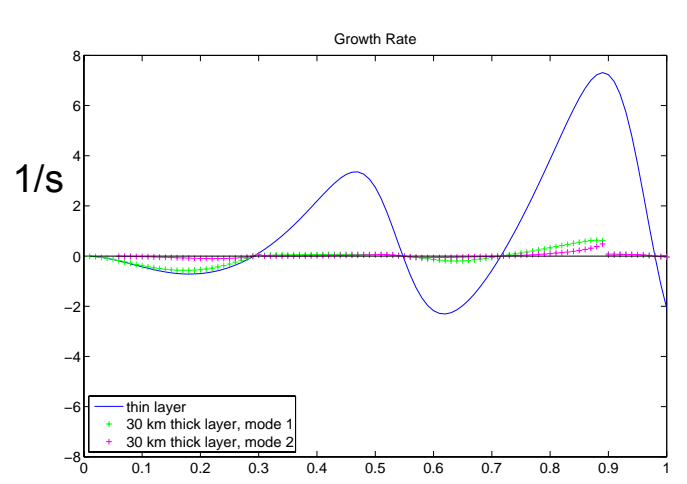

(a) $\mathrm{Hz}$

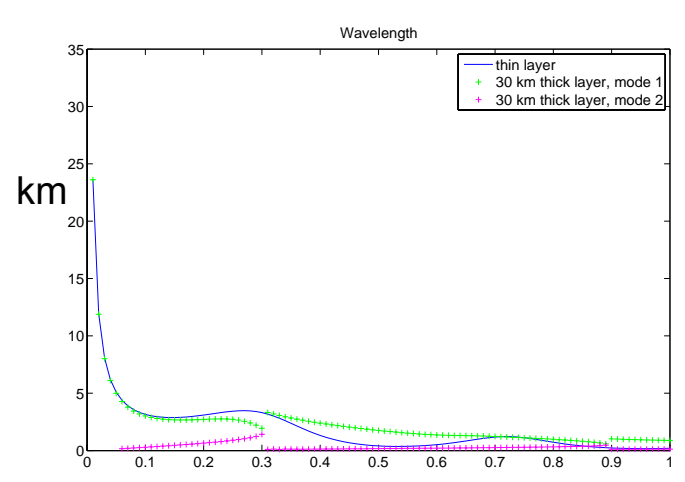

(b) $\mathrm{Hz}$

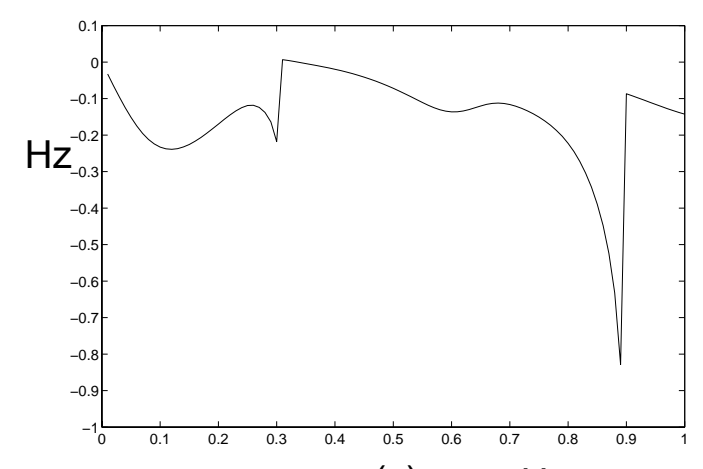

(c) $\mathrm{Hz}$

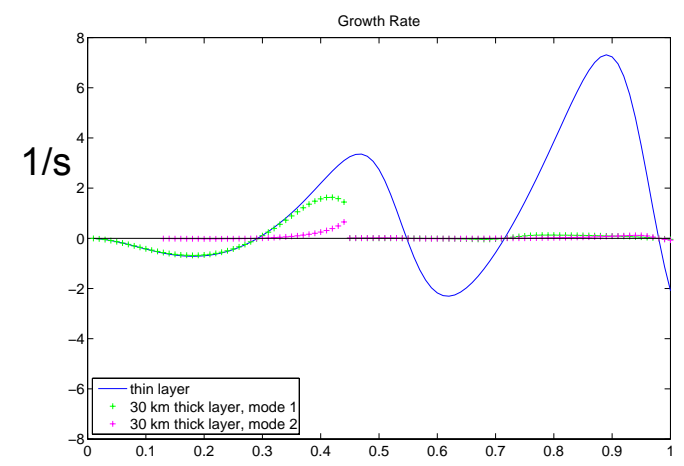

(d) $\mathrm{Hz}$

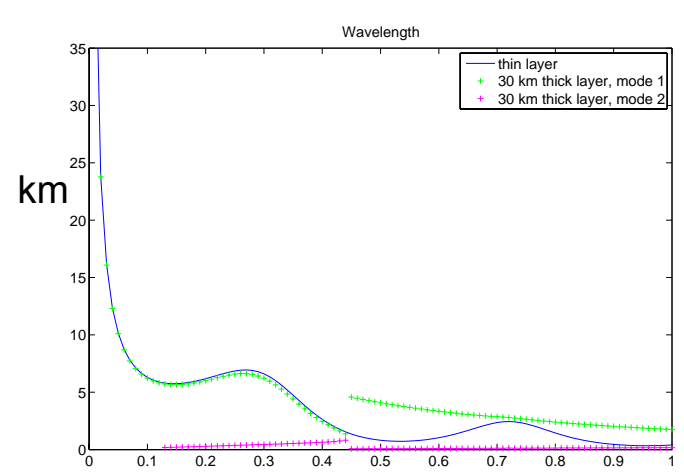

(e) $\mathrm{Hz}$

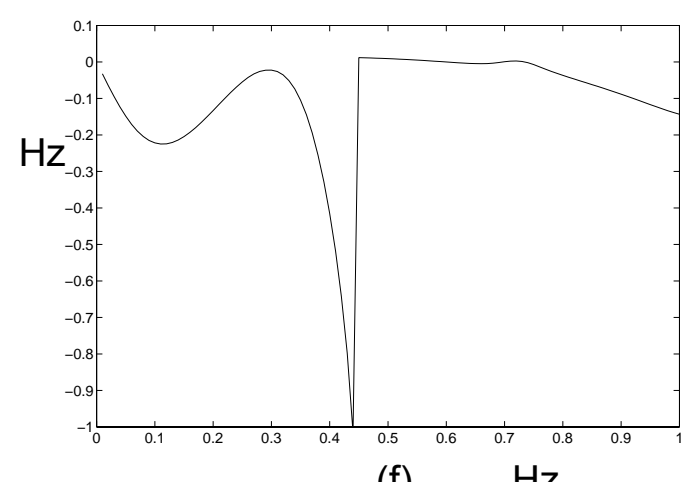

(f) $\quad \mathrm{Hz}$

Fig. 6. Growth rate, wavelength, and frequency in the earth-fixed frame, plotted versus frequency in the frame moving with the $\boldsymbol{E}_{0} \times \boldsymbol{B}$ velocity, for a $120 \mathrm{~km}$ altitude layer. Panels (a) through (c) are for $\boldsymbol{E}_{0}=50 \mathrm{mV} \mathrm{m}^{-1}$. Panels (d) through (f) are for $\boldsymbol{E}_{0}=100 \mathrm{mV} \mathrm{m}^{-1}$. The green (purple) lines show results for the mode with the largest (second largest) growth rate for a $30 \mathrm{~km}$ thick layer. The blue lines show results for a thin auroral layer of the same integrated conductivity. The results for the frequency in the earth-fixed frame (black lines) are shown only for the mode with the largest growth rate when the layer is $30 \mathrm{~km}$ thick (i.e., for the green lines). The integrated Pedersen and Hall conductivities are 2.27 mhos and 9.05 mhos, respectively, which corresponds to a $30 \mathrm{~km}$ thick layer with an electron density of $10^{11} \mathrm{~m}^{-3}$.

The growth rate above cutoff is typically an order of magnitude less than the growth rate below cutoff. The frequency of the cutoff increases with the background electric field. For the lower background electric field $\left(50 \mathrm{mV} \mathrm{m}^{-1}\right)$ two cutoffs are visible (first and second eigenmodes), with the first being below the lowest frequency thin-layer growth rate maxima. This results in the actual maximum growth rate being an order of magnitude less than the growth rate at the low- est frequency thin-layer growth rate maxima (which is in the vicinity of $0.5 \mathrm{~Hz}$ ). For the higher background electric field $\left(100 \mathrm{mV} \mathrm{m}^{-1}\right)$ the first cutoff is only a little below the lowest frequency thin-layer growth rate maxima, such that the actual maximum growth rate is $30 \%$ to $50 \%$ (depending on altitude, through $\kappa_{i}$ and $\kappa_{e}$ ) of the growth rate at the lowest frequency thin-layer growth rate maxima, with a $0.1 \mathrm{~Hz}$ reduction in the frequency of the maxima. 

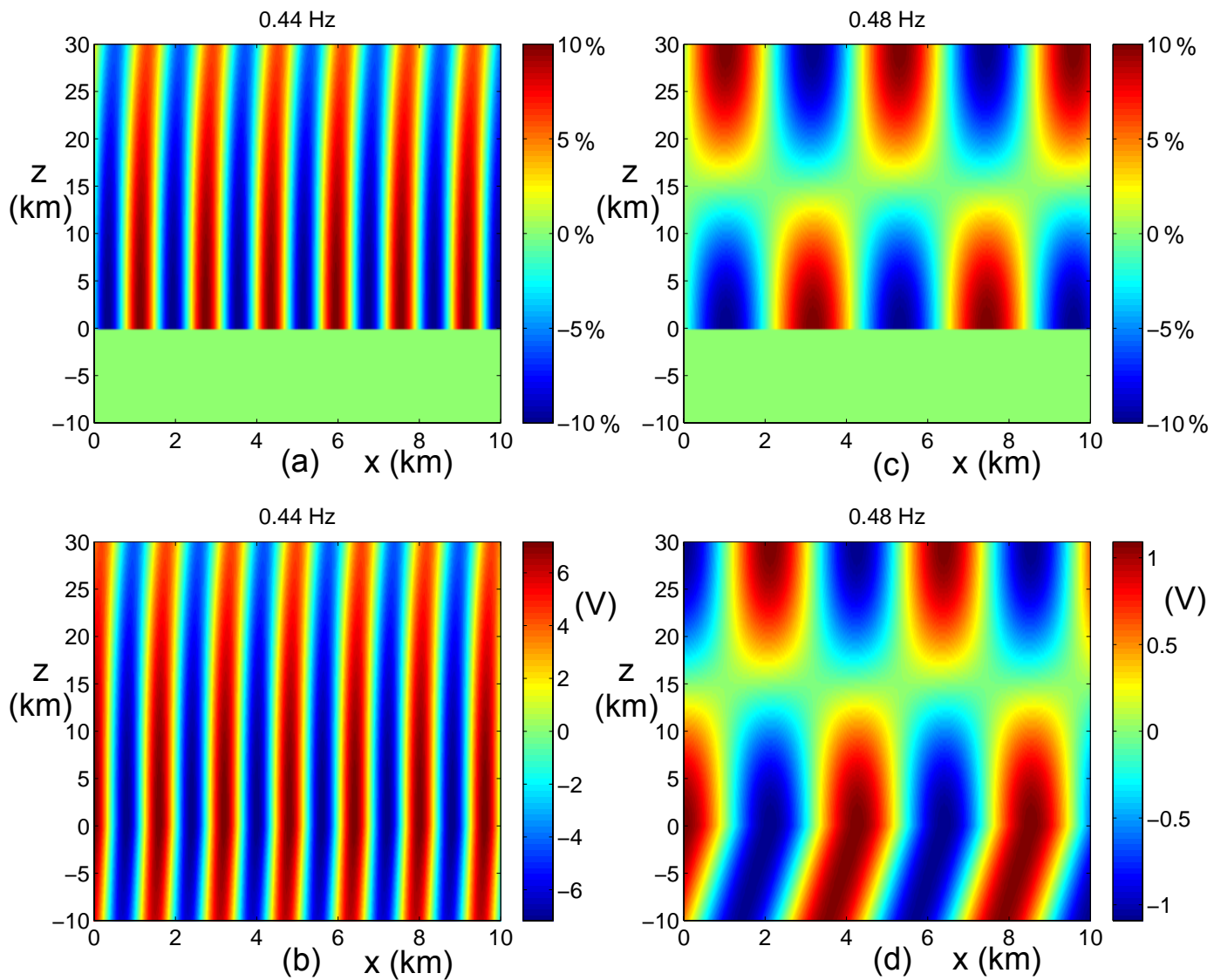

Fig. 7. Comparison of density and potential eigenfunctions below and above cutoff of the thin-layer mode, for $120 \mathrm{~km}$ altitude layer with $100 \mathrm{mV} \mathrm{m}^{-1}$ electric field. Panel (a) shows the percentage density perturbation for the mode with the highest growth rate just below cutoff (the thin-layer mode). Panel (b) shows the associated potential function for the thin-layer mode just below cutoff. Panel (c) shows the percentage density perturbation just above cutoff, for the remaining mode with the highest growth rate. Panel (d) shows the associated potential function just above cutoff, for the remaining mode with the highest growth rate.

The wavelength just before cutoff is, in three of the four examples, reduced by about $50 \%$ as compared to the thinlayer value. In all four cases the wavelengths at the growth rate maxima are quite short, being between one and three $\mathrm{km}$; in only one of the three cases did the wavelength exceed two $\mathrm{km}$.

Careful examination of the figures also shows deviations from the above generalizations of the behavior. For example, Fig. $5 \mathrm{c}$ and d shows a case where the growth rate at $0.9 \mathrm{~Hz}$ is the same as the growth rate at the lower frequency maxima (at about $0.4 \mathrm{~Hz}$ ), and the wavelength is also almost the same. Also, Figs. 6a and 6b show a case where the growth rate maximizes at $0.9 \mathrm{~Hz}$, although the wavelength is less than one $\mathrm{km}$.

The frequency axis in all the plots discussed above refers to the reference frame moving with the $\boldsymbol{E}_{0} \times \boldsymbol{B}_{0}$ drift. The results are especially simple in this reference frame. For example, the thin-layer growth rate is independent of the background electric field ( $\boldsymbol{E}_{0}$ in the earth fixed frame, which becomes the wind $\boldsymbol{u}$ in the $\boldsymbol{E}_{0} \times \boldsymbol{B}_{0}$ drift frame); this can be seen by solving the thin-layer dispersion relation (27) for the imaginary part of $\omega$ in terms of the real part of $\omega$. However, this independence certainly does not hold for a plot of the growth rate versus either wavelength, or frequency in the earth fixed frame. As expected, either of these plots would reveal the profound dependence of the growth rate on the background electric field. However, these plots are far more complex in appearance and we elect not to present them.

Figures 5c, 5f, 6c, and 6f show the dependence of the frequency in the earth-fixed frame on the frequency in the $\boldsymbol{E}_{0} \times \boldsymbol{B}_{0}$ frame (i.e., frame with $\boldsymbol{E}_{0}=0$ ), for the largest growth rate mode, as determined by a simple Doppler shift. It is negative because the Doppler shift causes the wave to reverse directions. Examination of the figures shows that the frequency of the unstable waves extends over a much larger range in the earth-fixed frame, and can include frequencies down to dc.

Although the layer thickness has been found to profoundly reduce the growth rate in many cases, as compared to the thin-layer case, the results still find very significant growth 

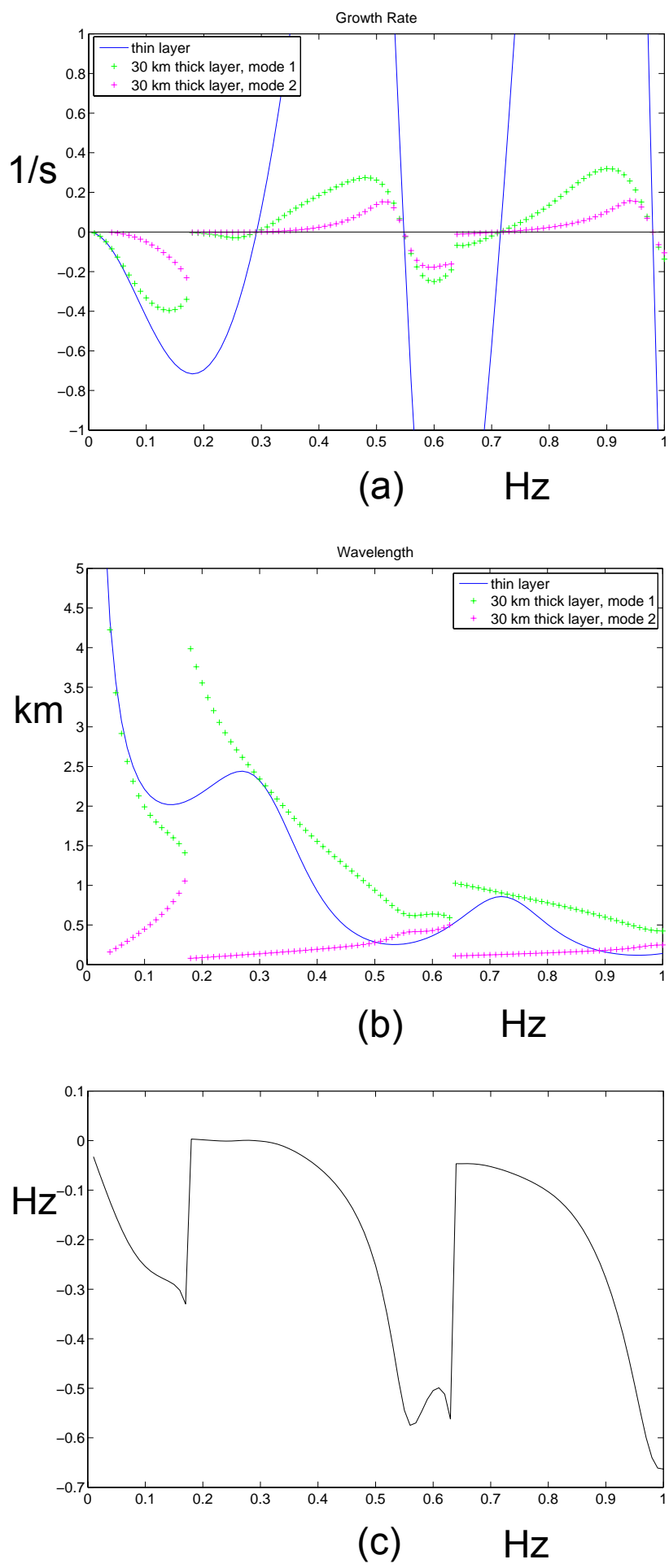

Fig. 8. Same as Figs. 6 a through $6 \mathrm{c}$, except for $\boldsymbol{E}_{0}=35 \mathrm{mV} \mathrm{m}^{-1}$, and with a finer vertical scale.

rates for rather moderate background electric fields. Figure 8 shows results for a $35 \mathrm{mV} \mathrm{m}^{-1}$ background electric field, with magnified axes. The maximum growth rate has an e-folding time of only $3 \mathrm{~s}$ ! This mode has a wavelength of $1 \mathrm{~km}$ and a frequency of $0.2 \mathrm{~Hz}$ in the earth-fixed frame. There is a positive growth rate down almost to $\mathrm{dc}$ in the earth fixed frame, where the wavelength exceeds $2.5 \mathrm{~km}$. There is also an unstable harmonic with a $3 \mathrm{~s}$ e-folding time, $0.5 \mathrm{~km}$ wavelength, and earth-fixed frequency of $0.4 \mathrm{~Hz}$.

The last figure we will discuss, Fig. 9, compares results with $\boldsymbol{E}_{0}$ pointing in the $\hat{y}$ direction (panels a-c), and $\boldsymbol{E}_{0}$ pointing in the $\hat{x}$ direction (panels $\mathrm{d}-\mathrm{f}$ ). In the latter case $\boldsymbol{E}_{0}$ points along the wavevector, which means that the relevant zeroth order current is determined by the Pedersen conductivity, and the Hall conductivity has no effect. In the former case, and all other cases discussed above, the reverse is true.

Figure 9 uses an extremely large electric field $\left(\boldsymbol{E}_{0}\right)$ of $250 \mathrm{mV} \mathrm{m}^{-1}$. A quick survey of data from many years operation of the Sondrestrom radar reveals that this is probably close to the largest value that has been observed in the Fregion ionosphere. This value was chosen to facilitate comparison with results in Streltsov and Lotko (2004).

Figure $9 \mathrm{a}-\mathrm{c}$ shows that the large $250 \mathrm{mV} \mathrm{m}^{-1}$ electric field overcomes the effect of the $30 \mathrm{~km}$ layer thickness to produce a growth rate that is $80 \%$ of the thin-layer growth rate (for the first harmonic). However, this only holds when the electric field is directed perpendicular to the wavevector, when the zeroth order current is a Hall current. Figure $9 \mathrm{~d}-\mathrm{f}$ shows that when the electric field is along the wavevector, the $30 \mathrm{~km}$ thickness of the layer reduces the growth rate by an order of magnitude from the thin-layer case.

This effect is summarized by noting that the role of the driving winds in the dispersion relation, that is, in Eqs. (25) and (26), can be consolidated into a single quantity, $u^{\prime}=$ $u_{\mathrm{x}}-\kappa_{i} u_{\mathrm{y}}$. The y-component of the wind, $u_{\mathrm{y}}$, is less effective than the $\mathrm{x}$-component, $u_{\mathrm{x}}$, by the factor $\kappa_{i}<1$. This can be understood by noting that the current along the wavevector drives the instability by polarizing the density perturbations associated with the wave: $u_{\mathrm{x}}$ drives a Hall current along the wavevector, while $u_{\mathrm{y}}$ drives a Pedersen current along the wavevector, and the Hall conductivity is greater than the Pedersen conductivity (in the altitude range under consideration). However, at higher altitudes the Pedersen conductivity becomes larger than the Hall conductivity, so that it should be most effective to position the background electric field along the wavevector.

\section{Discussion}

Linear analysis of instabilities is generally thought to give a good benchmark for evaluating the threshold for instability, and the wavelengths that are unstable. Therefore, the fact that the growth rate computed by assuming the ionosphere is infinitely thin can vary by an order of magnitude from the growth rate calculated without this assumption casts doubt on simulations and other analysis that utilize the thin-layer assumption. 


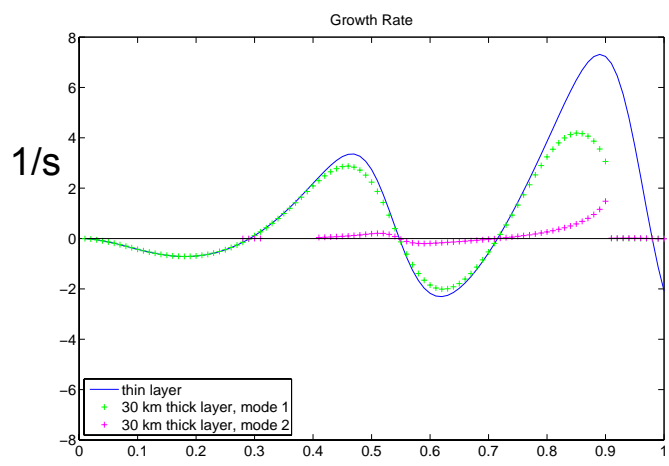

(a)

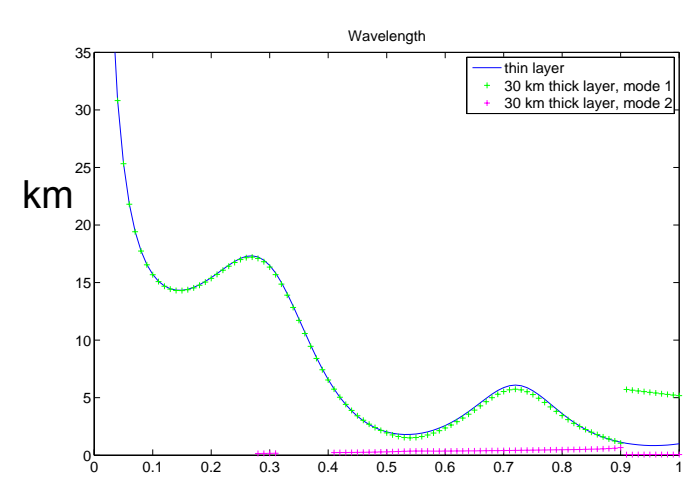

(b) $\mathrm{Hz}$

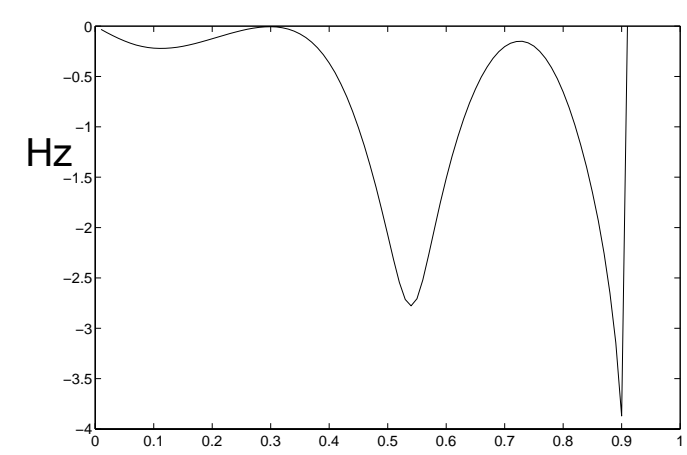

(c)

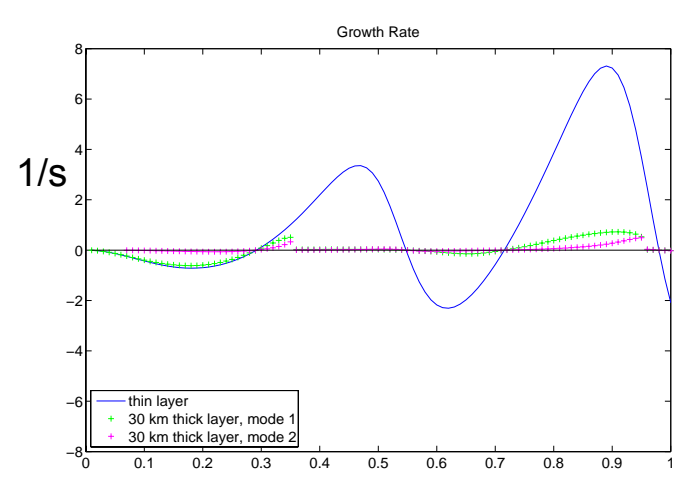

(d) $\mathrm{Hz}$

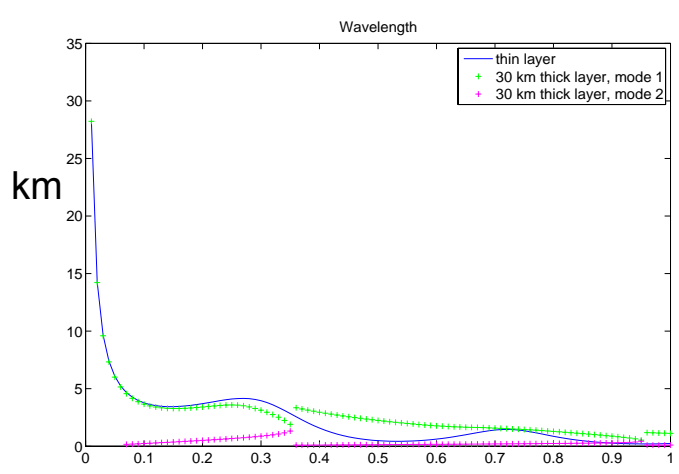

(e) $\mathrm{Hz}$

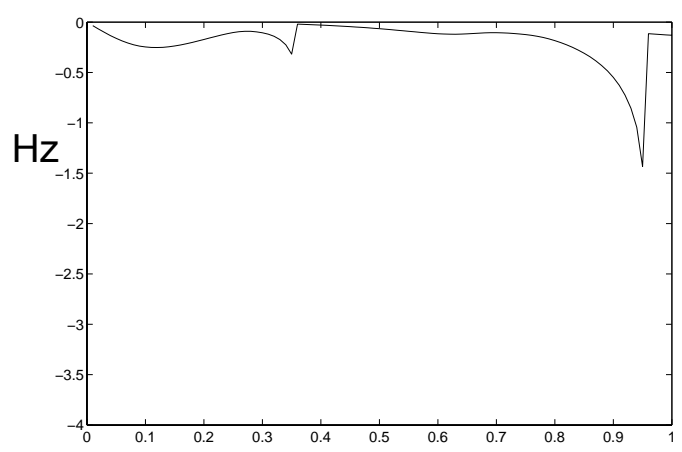

(f) $\quad \mathrm{Hz}$

Fig. 9. Comparison of results for $\boldsymbol{E}_{0}$ pointing perpendicular to the wavevector (panels a-c), and for $\boldsymbol{E}_{0}$ pointing along the wavevector (panels d-f). In all panels $\boldsymbol{E}_{0}=250 \mathrm{mV} \mathrm{m}^{-1}$. Growth rate, wavelength, and frequency in the earth-fixed frame, are plotted versus frequency in the frame moving with the $\boldsymbol{E}_{0} \times \boldsymbol{B}$ velocity, for a $120 \mathrm{~km}$ altitude layer. The green (purple) lines show results for the mode with the largest (second largest) growth rate for a $30 \mathrm{~km}$ thick layer. The blue lines show results for a thin auroral layer of the same integrated conductivity. The results for the frequency in the earth-fixed frame (black lines) are shown only for the mode with the largest growth rate when the layer is $30 \mathrm{~km}$ thick (i.e., for the green lines). The integrated Pedersen and Hall conductivities are 2.27 mhos and 9.05 mhos, respectively, which corresponds to a $30 \mathrm{~km}$ thick layer with an electron density of $10^{11} \mathrm{~m}^{-3}$.

Nevertheless, the growth rates calculated above are still quite substantial. For example, in Fig. 8 we found an efolding time of $3 \mathrm{~s}$, for an unstable wave with a frequency $0.2 \mathrm{~Hz}$ (in the earth-fixed frame), and a wavelength of $1 \mathrm{~km}$, when the effective background electric field is $35 \mathrm{mV} / \mathrm{m}$. Unless halted by other effects, one would expect that such a wave would grow to significant amplitude in a couple of min- utes. On the other hand, the wavelengths of these waves are quite short, rarely exceeding a few kilometers.

In the example just mentioned, the unstable wave does not involve the thin-layer IFI mode, but rather involves the second eigenmode. This eigenmode is of the type shown in Figs. 7c and 7d, which supports a parallel electric field. Therefore, unless some effect not considered stops 
the growth of these waves, they may contribute to electron heating and plasma structure in the auroral E-region ionosphere. Such E-region heating is normally attributed to Farley-Buneman waves, but it is difficult to account for the entire observed effect (e.g., Bahcivan and Cosgrove, 2010).

One effect that is not included in our analysis is recombination. Semeter and Kamalabadi (2005) give the recombination time scale for a variety of densities and altitudes. For the relatively low density of $10^{11} \mathrm{~m}^{-3}$ considered here, the recombination time scale exceeds $10 \mathrm{~s}$ at all altitudes. Hence, recombination should be negligible for frequencies greater than one $\mathrm{Hz}$ (one second period), and should be only a minor effect for the one half $\mathrm{Hz}$ frequency (two second period) that is of primary importance in this work.

Another effect that is not included in our analysis is any precipitation that may be caused by the Alfvén waves propagating away from the ionosphere and up to the auroral acceleration region. Alfvén waves in the inertial regime, which support a parallel electric field, can accelerate electrons such that they precipitate into the ionosphere (e.g., Hasegawa and Chen, 1975; Hasegawa, 1976; Goertz and Boswell, 1979; Ergun et al., 2005). The associated ionospheric density modulations could interact with the feedback mechanism. While we do not wish to assert that this effect is unimportant, we do note that modulated precipitation will only modulate the ionospheric density to the extent that the recombination time scale is short compared to the modulation period (Cosgrove et al., 2010). As argued in the previous paragraph, for the frequencies and densities considered here, it is doubtful that the recombination time scale is short enough to produce a substantial effect.

Except where noted, these results include the effects of Hall conductivity. Maximum growth rate occurs when the effective electric field is perpendicular to the wavevector, so that the density structures are polarized by the Hall current. Because the Pedersen conductivity is generally much less than the Hall conductivity in the region below $120 \mathrm{~km}$, the growth rate is much less when the effective electric field is directed along the wavevector. However, the reverse would be true at higher altitudes (not addressed by this work), where the Pedersen conductivity is larger.

The simulations of Streltsov and Lotko (2003, 2004, 2008) place the effective electric field along the wavevector. However, the E-region altitude for these simulations is substantially higher than considered here, so that the Pedersen conductivity is larger. This may mean that the $250 \mathrm{mV} / \mathrm{m}$ background electric field achieved in these simulations is sufficient to validate the use of an infinitely thin ionosphere, such as for the case seen in Fig. 9a-b. However, we note that even in Fig. 9b, the growth rate maximizes for a wavelength of $3 \mathrm{~km}$, whereas the grid size indicated in Streltsov and Lotko (2004) is $1.8 \mathrm{~km}$ (i.e., a Nyquist of $3.6 \mathrm{~km}$ ). Therefore, the simulation space may not be capable of fully representing the classical IFI with any layer thickness; it may be only the longer wavelength modes below the growth rate maxima that are resolved.

Finally, we give some discussion of the effects of the simplifications needed to make the analytic solution tractable. The E-region feedback is basically a process where the absorption of waves from the magnetosphere causes ion (Pedersen) current in the ionosphere to close with field aligned electron current from the magnetosphere, creating density depletions and enhancements, which become polarized by the background E-region current, which polarization launches a wave back into the magnetosphere, which is reflected back to the ionosphere, with potential feedback. The ionospheric parameters that govern this process for a thin E-region can be gleaned from Eq. (27). The numerator $u_{\mathrm{x}}-\kappa_{i} u_{\mathrm{y}}$ is the background ion velocity along the wavevector, which can be thought of as the background driving source. The only other ionosphere-dependent term is $\frac{e \kappa_{i} N}{Y_{m} B_{0}}$, which determines the impedance match between the ionospheric Pedersen conductivity and the magnetospheric admittance. Although Eq. (27) is specialized to the lower E-region, with a thin ionosphere, it motivates the general concept of an instability governed by the impedance match between the ionosphere and magnetosphere, and by the strength of the background driving source, which is the ion velocity along the wavevector in the reference frame where $\boldsymbol{E}_{0}=0$.

Using a more realistic ionospheric profile would mean that the background source strength (the ion velocity in the direction along the wavevector), would be a function of altitude (because the ion-neutral collision frequency is a function of altitude). In addition, the impedance match between the ionospheric antenna and the magnetospheric transmission line would be effected. However, as long as there is a good impedance match, and the vertically integrated source strength is strong, the instability should remain - that is, as long as the thickness of the ionosphere is not excessive.

When the ionosphere acquires thickness, then the "electrical length" across it may become important. If the time constant for penetration of the electric field through the ionosphere is on the order of the period of the unstable mode (i.e., the E-region has non-negligible electrical thickness), then these unstable modes must involve variation of the electric field along the magnetic field, and there should be a cutoff effect as found in this work. The fact that we find this cutoff effect separately for the collision frequencies applicable at $100 \mathrm{~km}$ and $120 \mathrm{~km}$, suggests that effecting a smooth transition between between these two values should not remove the cutoff effect. The fact that we find this cutoff effect for a rectangular E-region density profile suggests that the effect will endure for a smoothly varying E-region profile, as long as the E-region has an effective electrical thickness that is non-negligible. However, this question is ripe for a numerical simulation. 


\section{Summary of Cconclusions}

The main conclusions from this work are as follows:

1. Analysis of the IFI in terms of field line integrated quantities frequently gives order of magnitude errors in the linear growth rate. In the lower E-region, for modes with wavevector parallel to the effective background electric field $\boldsymbol{E}_{0}^{\prime}$, large errors remain for $\boldsymbol{E}_{0}^{\prime}$ exceeding $250 \mathrm{mV} \mathrm{m}^{-1}$. For modes with wavevector perpendicular to $\boldsymbol{E}_{0}^{\prime}$ - which take advantage in Hall conductivity - large $\boldsymbol{E}_{0}^{\prime}$ can overcome the effects of layer thickness. At higher E-region altitudes not addressed by this work, where the Pedersen conductivity is increased, we expect $\boldsymbol{E}_{0}^{\prime}$ along the wavevector to become more effective than in the lower E-region.

2. The reduction in growth rate comes because the thinlayer mode cuts off at a certain frequency, above which the mode with the highest growth rate involves electric fields parallel to $\boldsymbol{B}$. The slab thickness subsumes a wavelength (Fig. 7). The parallel electric field implies enhanced electron heating.

3. This result casts doubt on any analysis or simulation of the IFI in terms of field line integrated quantities. However, use of the thin layer assumption may be valid for large background electric fields $\left(\boldsymbol{E}_{0}\right)$, and/or, for thin auroral arcs.

4. The unstable wavelengths do not exceed 6 or $7 \mathrm{~km}$, even when the background electric field is up to $100 \mathrm{mV} \mathrm{m}^{-1}$. The wavelength that maximizes the growth rate is typically between one and three kilometers. Larger wavelengths can be achieved with very large background electric fields.

5. The growth rate for these few-km wavelength modes can be quite large, with e-folding times of a few seconds or less. Therefore, it is not so easy to find a reason why they should not grow.

6. Combining these results: a numerical simulation of the IFI, to be realistic, should include the effects of ionospheric thickness, and should have sub-km resolution in the ionosphere. If lower E-region arcs are to be simulated, then wavevectors perpendicular to the background electric field should be accommodated.

\section{Appendix A}

\section{Fluid equations and electrostatic approximation}

This appendix shows how Eqs. (8) through (12) can be derived from the fluid equations and Maxwell's equations, using the quasineutrality and electrostatic approximations. The electrostatic approximation is tested for the relevant parameter range and shown to be valid.

The complete set of Maxwell's equations and fluid equations for a two species plasma (ions and electrons) are as follows (e.g., Chen, 1984):

$$
\begin{aligned}
\epsilon_{0} \nabla \cdot \boldsymbol{E} & =n_{i} q_{i}+n_{e} q_{e} \\
\nabla \times \boldsymbol{E} & =-\frac{\partial \boldsymbol{B}}{\partial t} \\
\nabla \cdot \boldsymbol{B} & =0 \\
\mu_{0}^{-1} \nabla \times \boldsymbol{B} & =n_{i} q_{i} \boldsymbol{v}_{i}+n_{e} q_{e} \boldsymbol{v}_{e}+\epsilon_{0} \frac{\partial \boldsymbol{E}}{\partial t} \\
m_{j}\left[\frac{\partial \boldsymbol{v}_{j}}{\partial t}+\left(\boldsymbol{v}_{j} \cdot \nabla\right) \boldsymbol{v}_{j}\right] & =q_{j}\left(\boldsymbol{E}+\boldsymbol{v}_{j} \times \boldsymbol{B}\right) \\
& -m_{j} v_{j n}\left(\boldsymbol{v}_{j}-\boldsymbol{u}\right), j=i, e \\
\frac{\partial n_{j}}{\partial t} & =-\nabla \cdot\left(n_{j} \boldsymbol{v}_{j}\right), j=i, e,
\end{aligned}
$$

where subscripts $i$ and $e$ refer to ions and electrons, respectively, $n$ denotes density, $q$ denotes charge, $\boldsymbol{v}$ denotes velocity, $\epsilon_{0}$ is the free space permittivity, $\mu_{0}$ is the free space permeability, and the other symbols are as defined above. We will use the form $\mathrm{e}^{i(\omega t-\boldsymbol{k} \cdot \boldsymbol{x})}$ in discussing the reduction of Eqs. (A1) through (A6) to a simplified set through approximation.

The form $\mathrm{e}^{i(\omega t-\boldsymbol{k} \cdot \boldsymbol{x})}$ provides that $\frac{\partial \boldsymbol{B}}{\partial t}=i \omega \tilde{\boldsymbol{B}}$ (where $\boldsymbol{B}=$ $\boldsymbol{B}_{0}+\tilde{B}$, and $\boldsymbol{B}_{0}=$ const.). Therefore Eq. (A2) gives $\tilde{B}=$ $\frac{i}{\omega} \nabla \times \boldsymbol{E}$, which provides that $\nabla \cdot \tilde{B}=\frac{i}{\omega} \nabla \cdot \nabla \times \boldsymbol{E}=0$. So Eq. (A3) is automatically satisfied.

The left hand side of Eq. (A5) becomes $m_{j} i\left(\omega+v k_{v}\right) \boldsymbol{v}_{j}$. This can be directly compared with the term $m_{j} v_{j n} \boldsymbol{v}_{j}$ on the right hand side. Using the typical scale sizes for the $E$ region ionosphere, and from the analysis above, we have $\omega \lesssim$ $2 \pi \mathrm{s}^{-1}$, and $v k_{v} \lesssim 2 \pi\left(1 \mathrm{kms}^{-1}\right)\left(1 \mathrm{~km}^{-1}\right)=2 \pi \mathrm{s}^{-1}$, whereas $v_{j n} \gtrsim 400 \mathrm{~s}^{-1}$. Therefore, the left hand side of Eq. (A5) is negligible, and Eq. (A5) becomes what is known as the steady state momentum equation:

$0=q_{j}\left(\boldsymbol{E}+\boldsymbol{v}_{j} \times \boldsymbol{B}\right)-m_{j} v_{j n}\left(\boldsymbol{v}_{j}-\boldsymbol{u}\right), j=i, e$.

If we take the cross product of Eq. (A7) with $q_{j} \boldsymbol{B}$, and add the result back to $m_{j} v_{j n}$ times Eq. (A7), the result, after some manipulation, is the equivalent form

$\frac{v_{j n}}{\Omega_{j}^{\prime}} \boldsymbol{v}_{j}+\frac{\Omega_{j}^{\prime}}{v_{j n}} \boldsymbol{v}_{j \perp}=\frac{1}{B}(\boldsymbol{E}+\boldsymbol{u} \times \boldsymbol{B})+\frac{1}{B^{2}} \frac{\Omega_{j}^{\prime}}{v_{j n}} \boldsymbol{E} \times \boldsymbol{B}+\frac{v_{j n}}{\Omega_{j}^{\prime}} \boldsymbol{u}$,
$j=i, e$,

where the symbol $\perp$ denotes the component perpendicular to $\boldsymbol{B}$, and where $\Omega_{j}^{\prime}=\frac{q_{j} B}{m_{j}}$ (the prime denotes that this is a signed gyrofrequency).

For the case $j=i$ (i.e., for ions), applying the lower Eregion approximation $\frac{\Omega_{i}^{\prime}}{v_{i n}} \ll 1$ makes the second terms on both sides negligible, which gives Eq. (8). 
For the case $j=e$ (i.e., for electrons), the ionospheric approximation $\frac{\left|\Omega_{e}^{\prime}\right|}{v_{e n}} \gg 1$ gives

$\boldsymbol{v}_{e \perp}=\frac{1}{B^{2}} \boldsymbol{E} \times \boldsymbol{B}$, and

$\boldsymbol{v}_{e \|}=\frac{-\kappa_{e}}{B} \boldsymbol{E}_{\|}+\boldsymbol{u}_{\|}$.

where $\perp$ and $\|$ indicate the components perpendicular and parallel to $\boldsymbol{B}$. Subtracting Eq. (A9) from Eq. (8), and using the quasineutrality assumption $n=n_{i} \cong n_{e}$, we can calculate the current:

$$
\begin{aligned}
\boldsymbol{J}_{\perp} & =n e \boldsymbol{v}_{i \perp}-n e \boldsymbol{v}_{e \perp} \\
& =n e \frac{-\kappa_{i}}{B}\left(-\boldsymbol{E}_{\perp}-\boldsymbol{u} \times \boldsymbol{B}\right)+n e \frac{1}{B^{2}}\left(-\boldsymbol{E} \times \boldsymbol{B}+\boldsymbol{u}_{\perp} B^{2}\right), \\
J_{\|} & =n e v_{i \|}-n e v_{e \|}=n e\left(\frac{\kappa_{i}}{B}+\frac{\kappa_{e}}{B}\right) E_{\|} \cong n e \frac{\kappa_{e}}{B} E_{\|}, \quad(\mathrm{A} 10)
\end{aligned}
$$

where $e$ is the absolute value of $q_{j}$. With $\boldsymbol{E}=\boldsymbol{E}_{0}-\nabla \phi$, and $\boldsymbol{B}=-B_{0} \hat{z}$, these are exactly Eqs. (9) and (10).

Multiplying Eqs. (A6) by $q_{j}$, and adding the result for the case $j=i$ to the result for the case $j=e$ gives

$e \frac{\partial\left(n_{i}-n_{e}\right)}{\partial t}=\nabla \cdot\left(e n_{e} \boldsymbol{v}_{e}-e n_{i} \boldsymbol{v}_{i}\right)=-\nabla \cdot \boldsymbol{J}$.

Without any loss in generality, Eq. (A11) can replace one of the continuity Eqs. (A6), for example it can replace continuity for $j=e$. Using the quasineutrality assumption $n_{i} \cong n_{e}$, Eq. (A11) becomes

$\nabla \cdot \boldsymbol{J}=0$.

Given our assumption that all derivatives with respect to $y$ vanish, Eq. (A12) is equivalent to Eq. (11).

The assumption $\boldsymbol{E}=\boldsymbol{E}_{0}-\nabla \phi$ used in Eqs. (8) through (12) provides for a solution with $\nabla \times \boldsymbol{E}=0$, which does not satisfy Eq. (A2) exactly; this is known as the electrostatic assumption. Similarly, by using Eq. (11) we have not satisfied (A11) exactly; this known as the quasineutrality assumption. To show that these two assumptions give good approximate solutions to the full Eqs. (A1) through (A6), we should take the solutions obtained from Eqs. (8) through (12) and plug them into Eqs. (A4) and (A1) to get the solutions for $\tilde{B}$ and the charge density $n_{i} q_{i}+n_{e} q_{e}$, respectively. Then these solutions should be plugged into the Eqs. (A2) and (A11), to verify that these are satisfied in an approximate sense. By "satisfied in an approximate sense," we mean that only insignificant modifications are necessary to the solutions of Eqs. (8) through (12) in order to exactly satisfy Eqs. (A2) and (A11).

The validity of the quasineutrality approximation is discussed in many textbooks, for example (Chen, 1984), and we will not bother to verify it further here. We will, however, validate the electrostatic assumption as follows: Using our results from above and substituting into Eq. (A2) gives

$\nabla \times \boldsymbol{E}=\hat{y}\left(\frac{\partial E_{x}}{\partial z}-i k_{x} E_{z}\right)=-i \omega \tilde{B}$, which says that the only $\tilde{B}$ component is $B_{y}$. We need to compare the size of $\omega B_{y}$ (i.e., $\nabla \times \boldsymbol{E}$ ) with $k_{x} E_{z}$, in order to see if setting $B_{y}$ to zero in (A13) would make any significant difference in our result for $E_{z}$. To do this we solve for $j_{z}$ using both Eqs. (A4) and (A10), and set the results equal. Ignoring the displacement current, from Eq. (A4) we get

$J_{z}=\frac{i k_{x}}{\mu_{0}} B_{y}$.

From Eq. (A10), keeping only first order terms, we get

$$
J_{z}=n_{0} e \frac{\kappa_{e}}{B_{0}} E_{z}+n_{0} e \frac{\kappa_{i}}{B_{0}} u_{x} B_{y}-n_{0} e \frac{1}{B_{0}^{2}} E_{0 x} B_{y} .
$$

Setting these equal and solving for $i \omega B_{y}$ gives

$$
-i \omega B_{y}=\nabla \times \boldsymbol{E}=\frac{-i \omega \mu_{0} B_{0} n_{0} e \kappa_{e}}{i k_{x} B_{0}^{2}-\mu_{0} B_{0} e \kappa_{i} u_{x}+\mu_{0} n_{0} e E_{0 x}} E_{z} .
$$

We substitute into this using the characteristic unstable wavelength of $1 \mathrm{~km}$, the lower E-region $\kappa_{e}$ of 100 , the density of $10^{11} \mathrm{~m}^{-3}$ used throughout, the maximal E-region electric field of $100 \mathrm{mV} / \mathrm{m}$, and the $2000 \mathrm{~m} / \mathrm{s}$ wind associated with transforming to a reference frame that eliminates a $100 \mathrm{mV} / \mathrm{m}$ electric field (where the signs of the last two are chosen to maximize the result for $|\nabla \times \boldsymbol{E}|)$, and find

$\nabla \times \boldsymbol{E}=-0.0064 k_{x} E_{z}$.

Comparing Eq. (A17) with Eq. (A13) shows that the percentage error in $E_{z}$ from assuming $\frac{\partial E_{x}}{\partial z}=i k_{x} E_{z}$ (the electrostatic assumption) is very small. Also, because the scale size for variations in the z-direction is larger than the scale size for variations in the x-direction, the percentage error in $E_{x}$ is even smaller.

Therefore, the electrostatic assumption is a good approximation for the lower E-region problem solved in this work, within the parameter range that we consider. In addition, we have shown that the working Eqs. (8) through (12) can be derived in good approximation from the full set of fluid equations together with Maxwell's equations (Eqs. A1 through A6).

\section{Appendix B}

\section{Collisional Alfvén wave dispersion relation}

To derive the dispersion relation for Alfvén waves in the presence of collisions, consider the case with no neutral wind, and zero background electric field. Then using the form $\mathrm{e}^{i(\omega t-\boldsymbol{k} \cdot \boldsymbol{r})}$, and keeping terms to first order in $\boldsymbol{v}_{j}$, Eq. (A5) becomes

$\left(v_{j n}+i \omega\right) \boldsymbol{v}_{j}-\Omega_{j} \boldsymbol{v}_{j} \times \hat{b}=\frac{q_{j}}{m_{j}} \boldsymbol{E}$, 
where $\hat{b}$ is a unit vector along the background magnetic field. Cross multiplying this by $\hat{b}$ gives

$\left(v_{j n}+i \omega\right) \boldsymbol{v}_{j} \times \hat{b}+\Omega_{j} \boldsymbol{v}_{j}=\frac{q_{j}}{m_{j}} \boldsymbol{E} \times \hat{b}$.

Multiplying the first equation by $v_{j n}+i \omega$, the second equation by $\Omega_{j}$, adding them together, and solving for $\boldsymbol{v}_{j}$ gives

$\boldsymbol{v}_{j}=\frac{q_{j}}{m_{j}} \frac{\left(v_{j n}+i \omega\right) \boldsymbol{E}+\Omega_{j} \boldsymbol{E} \times \hat{b}}{\left(v_{j n}+i \omega\right)^{2}+\Omega_{j}^{2}}$.

For the electrons we can make the approximation $v_{e n}, \omega \ll$ $\Omega_{e}$, which gives

$\boldsymbol{v}_{e \perp}=\frac{1}{B} \boldsymbol{E} \times \hat{b}$.

Using Eqs. (B3) and (B4) we can compute the perpendicular part of the current as

$$
\begin{aligned}
\boldsymbol{J}_{\perp} & =n e \boldsymbol{v}_{i \perp}-n e \boldsymbol{v}_{e \perp}=\sigma_{P} \boldsymbol{E}_{\perp}-\sigma_{H} \boldsymbol{E} \times \hat{b}, \text { where } \\
\sigma_{P} & =\frac{n e^{2}}{m_{i}} \frac{v_{i n}-i \omega}{\left(v_{i n}-i \omega\right)^{2}+\Omega_{i}^{2}}, \text { and } \\
\sigma_{H} & =\frac{n e}{B} \frac{\left(v_{i n}-i \omega\right)^{2}}{\left(v_{i n}-i \omega\right)^{2}+\Omega_{i}^{2}} .
\end{aligned}
$$

Neglecting the displacement current, Eqs. (A4), (A2), and (B5) give

$\mu_{0}\left(\sigma_{P} \tilde{E}_{\perp}-\sigma_{H} \tilde{E} \times \hat{b}\right)=[-i \boldsymbol{k} \times \tilde{B}]_{\perp}, i \omega \tilde{B}=i \boldsymbol{k} \times \tilde{E}$,

where $\tilde{E}$ and $\tilde{B}$ are the wave electric and magnetic fields, respectively. Letting $\boldsymbol{k}=k_{\|} \hat{z}+k_{\perp} \hat{x}$, and ignoring the parallel electric field, we can eliminate $\tilde{B}$ between the equations in Eq. (B6) to obtain

$$
\begin{aligned}
& -i \omega \mu_{0}\left(\sigma_{P} E_{x}-\sigma_{H} E_{y}\right)=k_{\|}^{2} E_{x}, \\
& -i \omega \mu_{0}\left(\sigma_{P} E_{y}+\sigma_{H} E_{x}\right)=k^{2} E_{y} .
\end{aligned}
$$

Solving this system and writing $k_{\|}=k \cos \theta$ leads to the dispersion relation

$k^{4} \cos ^{2} \theta+i \omega \mu_{0} \sigma_{P}\left(1+\cos ^{2} \theta\right) k^{2}-\omega^{2} \mu_{0}^{2}\left(\sigma_{P}^{2}+\sigma_{H}^{2}\right)=0$,

which is a quadratic equation in $k^{2}$. Solving this quadratic equation, taking the negative sign for the shear mode, and substituting the expressions for $\sigma_{P}$ and $\sigma_{H}$ gives Eq. (6).

Acknowledgements. This material is based upon work supported by the National Science Foundation under Grant No. 0544750. The authors thank Bob Lysak for help deriving the collisional Alfvén wave dispersion relation.

Topical Editor K. Kauristie thanks two anonymous referees for their help in evaluating this paper.

\section{References}

Atkinson, G.: Auroral arcs: Result of the interaction of a dynamic magnetosphere with the ionosphere, J. Geophys. Res., 75(25), 4746, 1970.

Bahcivan, H. and Cosgrove, R.: On the generation of large wave parallel electric fields responsible for electron heating in the high-latitude $E$ region, J. Geophys. Res., in press, 2010.

Basu, B.: Characteristics of electromagnetic Rayleigh-Taylor modes in nighttime equatorial plasma, J. Geophys. Res., 110, A02303, doi:10.1029/2004JA010659, 2010.

Chaston, C. C., Bonnell, J. W., Carlson, C. W., Berthomier, M., Peticolas, L. M., Roth, I., and McFadden, J. P.: Electron acceleration in the ionospheric Alfvén resonator, J. Geophys. Res., 107(A11), 1413, doi:10.1029/2002JA009272, 2002.

Chen, F.: Introduction to plasma physics and controlled fusion, Second edition, Volume 1: Plasma physics, Plenum Press, New York, 1984.

Collin, R. E.: Foundations for microwave engineering, Physical and quantum electronics series, McGraw-Hill, 1966.

Cosgrove, R., Nicolls, M., Dahlgren, H., Ranjan, S., Sanchez, E., and Doe, R.: Radar detection of a localized $1.4 \mathrm{~Hz}$ pulsation in auroral plasma, simultaneous with pulsating optical emissions, and Pi1B magnetic pulsations, at substorm onset, Ann. Geophys., in review, 2010.

Ergun, R. E., Andersson, L., Su, Y. J., Newman, D. L., Goldman, M. V., Lotko, W., Chaston, C. C., and Carlson, C. W.: Localized parallel electric fields associated with inertial Alfvén waves, Phys. Plasmas, 12, 072901, doi:10.1063/1.1924495, 2005.

Goertz, C. K. and Boswell, R. W.: Magnetosphere-Ionosphere Coupling, J. Geophys. Res., 84(A12), 7239-7246, 1979.

Hasegawa, A. and Chen, L.: Kinetic process of plasma heating due to Alfvén wave excitation, Phys. Rev. Let., 35(6), 370-373, 1975.

Hasegawa, A.: Particle acceleration by MHD surface wave and formation of aurora, J. Geophys. Res., 81(28), 5083-5090, 1976.

Janhunen, P.: On the possibility of using an electromagnetic ionosphere in global MHD simulations, Ann. Geophys., 16, 397-402, doi:10.1007/s00585-998-0397-y, 1998.

Lysak, R. L.: Coupling of the dynamic ionosphere to auroral flux tubes, J. Geophys. Res., 91(A6), 7047-7056, 1986.

Lysak, R. L.: Feedback instability of the ionospheric resonant cavity, J. Geophys. Res., 96(A2), 1553-1568, 1991.

Mallinckrodt, A. J. and Carlson, C. W.: Relations between transverse electric fields and field-aligned currents, J. Geophys. Res. 83(A4), 1426-1432, 1978.

Maltsev, Y. P., Lyatsky, W. B., and Lyatskaya, A. M.: Currents over an auroral arc, Planet. Space. Sci., 25, 53-57, 1977.

Ossakow, S. L., Papadopoulos, K., Orens, J., and Coffey, T.: Parallel propagation effects on the type I electrojet instability, J. Geophys. Res., 80(1), 141-148, 1975.

Pokhotelov, O. A., Khruschev, V., Parrot, M., Senchenkov, S., and Pavlenko, V. P.: Ionospheric Alfvén resonator revisited: Feedback instability, J. Geophys. Res., 106(A11), 25813-25824, 2001.

Pokhotelov, D., Lotko, W., and Streltsov, A. V.: Simulations of resonant Alfvén waves generated by artificial HF heating of the auroral ionosphere, Ann. Geophys., 22, 2943-2949, doi:10.5194/angeo-22-2943-2004, 2004.

Sato, T.: A theory of quiet auroral arcs, J. Geophys. Res., 83(A3), 
1042-1048, 1978.

Semeter, J. and Kamalabadi, F.: Determination of primary electron spectra from incoherent scatter radar measurements of the auroral E region, Radio Sci., 40, 2, doi:10.1029/2004RS003042, 2005.

Streltsov, A. V. and Lotko, W.: Small-scale electric fields in downward auroral current channels, J. Geophys. Res., 108(A7), 1289, doi:10.1029/2002JA009806, 2003.

Streltsov, A. V. and Lotko, W.: Multiscale electrodynamics of the ionosphere-magnetosphere system, J. Geophys. Res., 109, A09214, doi:10.1029/2004JA010457, 2004.
Streltsov, A. V. and Lotko, W.: Coupling between density structures, electromagnetic waves and ionospheric feedback in the auroral zone, J. Geophys. Res., 113, A05212, doi:10.1029/2007JA012594, 2008.

Trakhtengertz, V. Y. and Feldstein, A. Y.: Quiet auroral arcs: ionospheric effect on magnetosphere convection stratification, Planet. Space. Sci., 32(2), 127-134, 1984. 